\title{
Index Modulation with Circularly-Shifted Chirps for Dual-Function Radar and Communications
}

\author{
Alphan Şahin, Safi Shams Muhtasimul Hoque, and Chao-Yu Chen
}

\begin{abstract}
In this study, we propose index modulation (IM) with circularly-shifted chirps (CSCs) (CSC-IM) for dual-function radar and communication (DFRC) systems. The proposed scheme encodes the information bits with the CSC indices and the phaseshift keying (PSK) symbols. It allows the receiver to exploit the frequency selectivity naturally in fading channels by combining IM and wideband CSCs. It also leverages the fact that a CSC is a constant-envelope signal to achieve a controllable peak-tomean envelope power ratio (PMEPR). For radar functionality, CSC-IM maintains the good autocorrelation (AC) properties of a chirp by ensuring that the transmitted CSCs are separated apart sufficiently in the time domain through index separation (IS). We investigate the impact of IS on spectral efficiency (SE) and obtain the corresponding mapping functions. For theoretical results, we derive the union bound (UB) of the block error rate (BLER) for arbitrary chirps and the Cramer-Rao lower bounds (CRLBs) for the range and reflection coefficients for the matched filter (MF)based estimation. We also prove that complementary sequences (CSs) can be constructed through CSCs by linearly combining the Fourier series of CSCs. Finally, through comprehensive comparisons, we demonstrate the efficacy of the proposed scheme for DFRC scenarios.
\end{abstract}

Index Terms-Chirps, complementary sequences (CSs), index modulation (IM), peak-to-mean envelope power ratio (PMEPR)

\section{INTRODUCTION}

The merging of communications and radar functionalities into a single wireless network can improve the efficient utilization of the physical resources and address the potential interference issues between radar and communication systems [3]. To realize such a network, the transmitted signals need to be designed based on two different objectives. For radar functionality, the primary goal is to improve the accuracy of the range and/or velocity estimations. On the other hand, the waveform for communications is optimized by considering communications-related metrics such as error rate or data rate. Hence, developing a transmission scheme suitable for both features is not trivial [4]. In this study, we address this issue and propose index modulation (IM) with circularlyshifted chirps (CSCs) (CSC-IM) for dual-function radar and communication (DFRC) systems, which can be synthesized with discrete Fourier transform spread orthogonal frequency division multiplexing (DFT-s-OFDM) used in 3GPP Fifth

Alphan Şahin and Safi Shams Muhtasimul Hoque are with the Electrical Engineering Department, University of South Carolina, Columbia, SC, USA Chao-Yu Chen is with Department of Engineering Science, National Cheng Kung University, Tainan, Taiwan, R.O.C. E-mails: asahin@mailbox.sc.edu, shoque@email.sc.edu, super@mail.ncku.edu.tw

This paper was presented in part at the IEEE Global Communications Conference - Workshop on Integrated Sensing and Communications 2020 [1] and IEEE Consumer Communications \& Networking Conference 2021 [2].
Generation (5G) New Radio (NR) [5] and Fourth Generation (4G) Long-Term Evolution (LTE) uplink [6].

Chirps are prominent for radar applications due to their excellent peak-to-mean envelope power ratio (PMEPR) and good autocorrelation (AC) properties in time-varying channels. They also facilitate the radar implementation with a simple hardware architecture through correlations in radio-frequency (RF). They were first proposed in [7] to achieve a long-range high-resolution radar, which was later extended to encode information through the slope of the chirps in the time-frequency (TF) plane. For developing more sophisticated multiplexing methods based on chirps, the bases constructed through chirps have been studied in the literature, extensively. For example, in [8], an orthogonal amplitude-variant linear chirp set where each chirp has a different chirp rate was proposed. In [9], orthogonal chirps were constructed by shifting the chirps in the frequency domain. In [10], CSCs were proposed by using the structure of DFT-s-OFDM with a specific frequency-domain spectral shaping (FDSS) function. It was shown that CSCs can be transmitted simultaneously in an overlapping manner as opposed to linear chirps. However, these studies do not particularly consider DFRC applications.

Another way of constructing a scheme suitable for DFRC is to utilize the multiplexing methods that are highly used in wireless communication systems. In [3], orthogonal frequency division multiplexing (OFDM), chirps, and complementary sequences (CSs) with single-carrier (SC) waveform and OFDM [11], [12] are surveyed for DFRC scenarios. It is emphasized that the noise-like nature of OFDM signals in time can be beneficial for a typical radar at the expense of high PMEPR and the sidelobe growth in the AC function due to the existence of cyclic prefix (CP). On the other hand, OFDM provides an excellent framework for the channel frequency response (CFR)-based estimation methods as the processing directly occurs in the modulation domain. For example, in [13], several range profiles were demonstrated through CFR by using OFDM as a radar waveform. In [14], OFDM is compared with chirps and other spread-spectrum techniques in detail. In [15] and [16], maximum-likelihood (ML)-based range and velocity estimators for a single target are investigated for OFDM. An iterative algorithm based on filtering and clipping was investigated in [17] to reduce the PMEPR of the multicarrier radar waveform at a cost of the distorted AC function. In [18], the weighted subcarriers were proposed to mitigate the PMEPR for an OFDM-based radar. The authors in [19] proposed to transmit optimized complexvalued data through the unused subcarriers of OFDM for radar functionality. In [20], a real-valued baseband OFDM 
signal that modulates the phase of the carrier, called constantenvelope OFDM, was proposed for detecting a target in a multi-path scenario. In [21], the IEEE 802.11ad SC preamble based on CSs was exploited for DFRC applications.

Recently, IM receives attention for DFRC scenarios as it promises communications with minimal degradation to the radar performance [4]. IM is a permutation modulation [22] and encodes the information in the order of discrete objects, e.g., antennas, subcarriers, or time slots. For a comprehensive survey on IM and its applications, we refer the reader to the surveys in [23]-[26] and the references therein. For communications applications, in [27], IM with OFDM (OFDM-IM) is investigated by grouping the subcarriers and activating or deactivating subcarriers within the groups for encoding information. In [28], extra information bits were transmitted by turning on and off the antennas, i.e., spatial modulation. In [29], media-based modulation was proposed by setting the on/off status of available RF mirrors. For radar applications, in [30], the information was encoded by shuffling the radar signals for a multi-input multi-output (MIMO) radar setup. In [31], it was proposed to select a subset of subcarriers and/or antennas. In this study, with the same motivation of minimum degradation to radar, we utilize IM with CSCs.

\section{A. Contributions}

In this study, we provide both theoretical and practical contributions listed as follows:

- A scheme for DFRC systems: We propose to transmit multiple modulated CSCs simultaneously for a DFRC system, where the information bits are encoded with indices and phase-shift keying (PSK) symbols. Since it relies on the structure of DFT-s-OFDM with a special FDSS and IM, it leads to low-complexity transmitters and receivers. The main advantage of the proposed scheme is controllable low-PMEPR and spectral efficiency (SE) while still being a wideband signal needed for radar functionality at radar receiver $(\mathrm{RXr})$ and exploiting frequency selectivity at communication receiver $(\mathrm{RXc})$.

- Theoretical bounds and relationships: We establish a connection between CSs and chirps. We derive the union bound (UB) of block error rate (BLER) for the proposed scheme. Also, for radar functionality, we obtain the Cramer-Rao lower bounds (CRLBs) for ranges and reflection coefficients, which consider the phase of the reflected signal as a function of the target's range.

- Improved range estimation with Index Separation: We investigate two range estimation methods: Matched filter (MF)-based and linear minimum mean square error (LMMSE)-based estimations. To increase the estimation accuracy, we introduce a concept called index separation $(I S)$ that ensures a low AC zone. We derive the maximum separation between CSCs in time without affecting the SE for any number of chirps, theoretically. To facilitate the encoder and decoder with the IS, we also develop methods that construct a bijective mapping between the information bits and indices.

- Comprehensive comparisons: The proposed scheme is compared with OFDM-IM, IM with DFT-s-OFDM
(DFT-s-OFDM-IM), and the CSs based on Reed-Muller (RM) code (CSs-RM), comprehensively, in terms of PMEPR, error rate, estimation accuracy, and radar resolution in various scenarios, which provides further insights into DFRC waveform.

The rest of the paper is organized as follows. In Section III, the system model is provided. In Section [II] we introduce CSC-IM and derive the UB of BLER. The relationship between CSs and chirps and the trade-off between SE and PMEPR are discussed in this section. In IV we analyze the radar functionality with CSC-IM. We discuss range estimation and IS. In Section $\nabla$, numerical results are presented. The paper is concluded in Section VI.

\section{PREliminaries AND System MOdel}

The sets of complex numbers, real numbers, non-negative real numbers, positive integers, non-negative integers, and the set of integers $\{0,1, \ldots, H-1\}$ are denoted by $\mathbb{C}, \mathbb{R}, \mathbb{R}_{0}^{+}, \mathbb{Z}^{+}$, $\mathbb{Z}_{0}^{+}$, and $\mathbb{Z}_{H}$ respectively. Conjugation is denoted by $(\cdot)^{*}$. The notation $\left(a_{0}, a_{1}, \ldots, a_{M-1}\right)$ represents the sequence $\boldsymbol{a}$. The constant $\mathrm{j}$ denotes $\sqrt{-1}$.

\section{A. Circularly-Shifted Chirps}

Let $\psi_{0}(t) \in \mathbb{R}$ be a periodic function with the period of $T_{\mathrm{s}}$. We then define the function $B_{m}(t)$ by setting $B_{m}(t)=$ $\mathrm{e}^{\mathrm{j} \psi_{m}(t)}$, where $\psi_{m}(t)=\psi_{0}\left(t-\tau_{m}\right)$ and $\tau_{m}$ is the amount of translation for $m \in\{0,1, \ldots, M-1\}$ and $\tau_{0}=0$. The function $B_{m}(t)$ for $t \in\left[0, T_{\mathrm{s}}\right)$ is a CSC and it is equal to the circularly-shifted version of the function $B_{0}(t)$ by $\tau_{m}$ seconds.

The Fourier series expansion of $B_{m}(t)$ can be obtained as

$$
B_{m}(t) \approx \sum_{k=L_{\mathrm{d}}}^{L_{\mathrm{u}}} c_{k} \mathrm{e}^{\mathrm{j} 2 \pi k \frac{t-\tau_{m}}{T_{\mathrm{s}}}}
$$

where $L_{\mathrm{d}}<0$ and $L_{\mathrm{u}}>0$ are integers, and $c_{k}$ is the $k$ th Fourier coefficient given by

$$
c_{k}=\mathcal{F}\left\{\mathrm{e}^{\mathrm{j} \psi_{0}(t)}\right\} \triangleq \frac{1}{T_{\mathrm{s}}} \int_{T_{\mathrm{s}}} \mathrm{e}^{\mathrm{j} \psi_{0}(t)} \mathrm{e}^{-\mathrm{j} 2 \pi k \frac{t}{T_{\mathrm{s}}}} d t .
$$

Let $D / 2 T_{\mathrm{s}}$ denote the maximum frequency deviation around the carrier frequency. For an accurate approximation of the right-hand side of (1) to $B_{m}(t)$, we assume that $L_{\mathrm{d}}<-D / 2$ and $L_{\mathrm{u}}>D / 2$. This is due to the fact that $B_{m}(t)$ is a function where $\left|c_{k}\right|$ approaches zero rapidly for $|k|>D / 2$. Note that the actual bandwidth of a chirp is slightly larger than twice the maximum frequency deviation [32]. It can be calculated based on the total integrated power of the transmitted spectrum, i.e., occupied channel bandwidth (OCB). In this study, we express the OCB as $M_{\mathrm{ocb}} / T_{\mathrm{s}} \mathrm{Hz}$, where $M_{\mathrm{ocb}}$ is assumed to be a positive integer. Also, the instantaneous frequency of $B_{m}(t)$ around the carrier frequency $f_{\mathrm{c}}$ can be obtained as $F_{m}(t)=$ $\frac{1}{2 \pi} d \psi_{m}(t) / d t \mathrm{~Hz}$.

In [10], CSCs are utilized for data transmission by using DFT-s-OFDM as follows: Consider a baseband signal given by $x(t)=\frac{1}{\sqrt{M}} \sum_{m=0}^{M-1} d_{m} B_{m}(t)$, where $d_{m}$ denotes the $m$ th modulation symbol. If $\tau_{m}$ is chosen as $\tau_{m}=m / M \times T_{\mathrm{s}}$, i.e., uniform spacing in time, by sampling $x(t)$ at $n / N \times T_{\mathrm{s}}$ for 
$n \in\{0,1, \ldots N-1\}$ and using (1), the baseband signal $x(t)$ in discrete time can be written as

$$
x_{n}=\underbrace{\sum_{k=L_{\mathrm{d}}}^{L_{\mathrm{u}}} \underbrace{c_{k}}_{\text {-point IDFT with zero-padding }}}_{\text {Frequency-domain spectral shaping }},
$$

where $N>M=L_{\mathrm{u}}-L_{\mathrm{d}}+1>D$. Hence, by prepending a CP with the duration of $T_{\mathrm{CP}}$ to the signal in (3), a typical DFT-s-OFDM transmitter or receiver can be utilized for synthesizing modulated CSCs by only introducing a special FDSS filter, i.e., $\left\{c_{k} \mid k=L_{\mathrm{d}}, \ldots, L_{\mathrm{u}}\right\}$.

In [10], several closed-form expressions for $c_{k}$ are also provided. For example, let $F_{0}(t)$ be a function changing from $-\frac{D}{2 T_{\mathrm{s}}}$ Hz to $\frac{D}{2 T_{\mathrm{s}}} \mathrm{Hz}$, i.e., $F_{0}(t)=\frac{D}{2 T_{\mathrm{s}}}\left(\frac{2 t}{T_{\mathrm{s}}}-1\right)$, i.e., a linear chirp. The $k$ th Fourier coefficient for the linear chirp can be calculated as

$$
c_{k}=\gamma_{k}\left(C\left(\alpha_{k}\right)+C\left(\beta_{k}\right)+\mathrm{j} S\left(\alpha_{k}\right)+\mathrm{j} S\left(\beta_{k}\right)\right),
$$

where $C(\cdot)$ and $S(\cdot)$ are the Fresnel integrals with cosine and sine functions, respectively, and $\alpha_{k}=(D / 2+2 \pi k) / \sqrt{\pi D}$, $\beta_{k}=(D / 2-2 \pi k) / \sqrt{\pi D}, \gamma_{k}=\sqrt{\frac{\pi}{D}} \mathrm{e}^{-\mathrm{j} \frac{(2 \pi k)^{2}}{2 D}-\mathrm{j} \pi k}$. For sinusoidal chirps, $F_{0}(t)=\frac{D}{2 T_{\mathrm{s}}} \cos \left(2 \pi \frac{t}{T_{\mathrm{s}}}\right)$ and it can be shown that

$$
c_{k}=J_{k}\left(\frac{D}{2}\right)
$$

where $J_{k}(\cdot)$ is the Bessel function of the first kind of order $k$.

\section{B. Complementary Sequences}

A sequence pair $(\boldsymbol{a}, \boldsymbol{b})$ of length $M$ is a Golay complementary pair (GCP) if the aperiodic autocorrelation functions (AACFs) of the sequences $\boldsymbol{a}$ and $\boldsymbol{b}$ sum to zero for all non-zero lags [33], i.e., $\rho_{\boldsymbol{a}}(l)+\rho_{\boldsymbol{b}}(l)=0$ for $l \neq 0$, where $\rho_{\boldsymbol{a}}(l)$ and $\rho_{\boldsymbol{b}}(l)$ are the AACFs of the sequences $\boldsymbol{a}$ and $\boldsymbol{b}$ at the $l$ th lag, respectively. The AACF of the sequence $\boldsymbol{a}$ can be calculated as

$$
\rho_{\boldsymbol{a}}(l) \triangleq \begin{cases}\sum_{i=0}^{M-l-1} a_{i}^{*} a_{i+l}, & 0 \leq l \leq M-1 \\ \sum_{i=0}^{M+l-1} a_{i} a_{i-l}^{*}, & -M+1 \leq l<0 . \\ 0, & \text { otherwise }\end{cases}
$$

Each sequence in a GCP is called a CS. A GCP $(\boldsymbol{a}, \boldsymbol{b})$ can equivalently be defined as any sequence pair satisfying $|A(z)|^{2}+|B(z)|^{2}=\rho_{\boldsymbol{a}}(0)+\rho_{\boldsymbol{b}}(0)$, where $A(z) \triangleq$ $a_{M-1} z^{M-1}+a_{M-2} z^{M-2}+\cdots+a_{0}$ and $B(z) \triangleq b_{M-1} z^{M-1}+$ $b_{M-2} z^{M-2}+\cdots+b_{0}$ in indeterminate $z$ [34].

Let $s_{\boldsymbol{a}}(t)=\sum_{i=0}^{M-1} a_{i} \mathrm{e}^{\mathrm{j} 2 \pi i \frac{t}{T_{\mathrm{s}}}}$ for $t \in\left[0, T_{\mathrm{s}}\right)$ be a continuous-time baseband OFDM symbol generated from a sequence $\boldsymbol{a}$ with the symbol duration $T_{\mathrm{s}}$. It can be shown that the instantaneous peak power of $s_{\boldsymbol{a}}(t)$ is bounded, i.e., $\max _{t}\left|s_{\boldsymbol{a}}(t)\right|^{2} \leq \rho_{\boldsymbol{a}}(0)+\rho_{\boldsymbol{b}}(0)$, if $\boldsymbol{a}$ is a CS. For this case, the PMEPR of the OFDM symbol $s_{\boldsymbol{a}}(t)$, defined as $\max _{t}\left|s_{\boldsymbol{a}}(t)\right|^{2} / P_{\mathrm{av}}$, is less than or equal to $10 \log _{10}(2) \approx 3 \mathrm{~dB}$

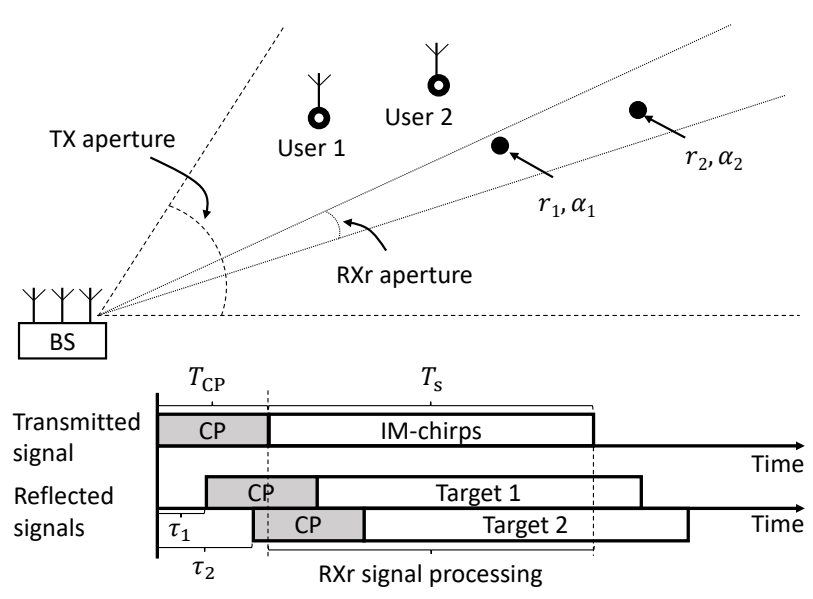

Fig. 1. DFRC scenario and the corresponding timing diagram for the transmitted signal and the radar return for two targets.

if $P_{\mathrm{av}}=\rho_{\boldsymbol{a}}(0)=\rho_{\boldsymbol{b}}(0)$ [35]. For non-unimodular CSs, $\rho_{\boldsymbol{a}}(0)$ may not be equal to $\rho_{\boldsymbol{b}}(0)$. In that case, the power of an OFDM symbol with $\boldsymbol{a}$ can be different from the one with $\boldsymbol{b}$ although the instantaneous peak power is still less than or equal to $\rho_{\boldsymbol{a}}(0)+\rho_{\boldsymbol{b}}(0)$. Hence, to avoid misleading results, we define $P_{\mathrm{av}}$ as the power of the entire baseband signal in this study.

\section{DFRC Scenario}

In this study, we consider a DFRC scenario where a base station (BS) broadcasts a communication signal to the users and exploits the same signal to estimate the distances of the surrounding objects as illustrated in Fig. 1. We assume that a directional antenna at the RXr sweeps along azimuth and elevation angles within the aperture of the transmitter (TX) to identify the orientation of the targets.

We consider a low-velocity environment, e.g., indoors, where the coherence time is much larger than $T_{\mathrm{s}}$. Hence, the channel impulse response (CIR) within $T_{\mathrm{S}}$ can be assumed to be time-invariant and expressed as $h(\tau)=$ $\sum_{s=1}^{R} \alpha_{s} \delta\left(\tau-\tau_{s}\right)$, where $R$ is the number of paths, and $\alpha_{s} \in \mathbb{R}$ and $\tau_{s} \in \mathbb{R}_{0}^{+}$are the gain and the delay of the path TX-to-sth target-to-RXr, respectively. The path delays can be calculated as $\tau_{s}=2 r_{s} / c$, where $r_{s} \in \mathbb{R}$ is the distance between the $s$ th target and the BS and $c$ is the speed of light. The BS's goal is to estimate $r_{s}$ for $s \in\{1, \ldots, R\}$ while using the same signal for broadcasting information.

We assume that the TX and RXr at the BS are synchronized in time and there is an ideal phase/frequency synchronization between the TX and RXr carriers (e.g., fed through the same oscillator). For an OFDM-based waveform, the CFR on the $k$ th subcarrier can be calculated as

$$
\zeta_{k}=\left.\int h(\tau) \mathrm{e}^{-\mathrm{j} 2 \pi f \tau} d \tau\right|_{f=f_{\mathrm{c}}+\frac{k}{T_{\mathrm{s}}}}=\sum_{s=1}^{R} \alpha_{s} \mathrm{e}^{-\mathrm{j} 2 \pi f_{\mathrm{c}} \tau_{s}} \mathrm{e}^{-\mathrm{j} 2 \pi k \frac{\tau_{s}}{T_{\mathrm{s}}}}
$$

where we assume that $\tau_{R} \leq T_{\mathrm{CP}}$ and the RXr processes the radar return for $t \in\left[T_{\mathrm{CP}}, T_{\mathrm{s}}+T_{\mathrm{CP}}\right)$ so that the path delays include the propagation delays. Therefore, the maximum range 


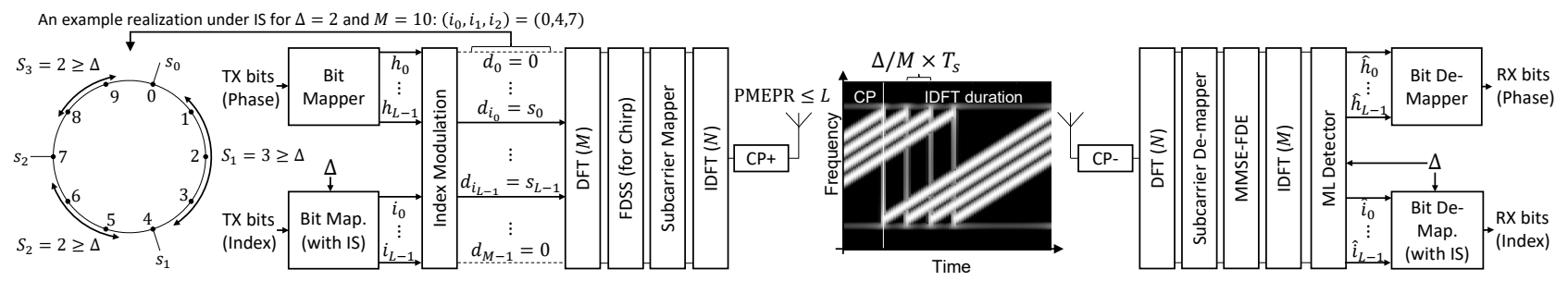

Fig. 2. Transmitter and receiver for the proposed scheme and an example realization under IS for $\Delta=2$ and $M=10$.

of the radar for this specific implementation is $c \times T_{\mathrm{CP}} / 2$ meters. Note that it is possible to increase the maximum range if the RXr's synchronization point is intentionally delayed and chosen based on energy detection or prior information related to the environment, at the cost of extra processing. It is also worth noting that the phase term in (7) is a function of target's location, which plays a major role for increasing the accuracy of range estimation as discussed in Section IV-C

\section{INDEX MODUlation With CiRCUlaRly-SHIFTED CHIRPS}

At the transmitter, $p$ information bits are first split into two groups: $p_{1}$ bits for choosing $L$ different CSCs from the set $\mathbb{W}=\left\{B_{m}(t) \mid m=0,1, \ldots, M-1\right\}$ and $p_{2}$ bits for $L$ different $H$-PSK symbols that are multiplied with the chosen CSCs. Let $\boldsymbol{i}=\left(i_{0}, i_{1}, \ldots, i_{L-1}\right)$ for $0 \leq i_{p}<i_{r}<M$ and $p<r$ and $\boldsymbol{h}=\left(h_{0}, h_{1}, \ldots, h_{L-1}\right)$ for $h_{\ell} \in \mathbb{Z}_{H}$ denote the indices of the chosen CSCs and the integers to be mapped to $H$ PSK symbols, respectively. Without loss of generality, the $\ell$ th $H$-PSK symbol can be calculated as $s_{\ell}=\mathrm{e}^{\mathrm{j} 2 \pi h_{\ell} / H}$ for $\ell \in$ $\{0,1, \ldots, L-1\}$. The baseband signal for the CSC-IM can be written as $x(t)=\frac{1}{\sqrt{L}} \sum_{\ell=0}^{L-1} s_{\ell} B_{i_{\ell}}(t)$. Therefore, by using (3), the discrete-time signal can be expressed as

$$
x_{n}=\sum_{k=L_{\mathrm{d}}}^{L_{\mathrm{u}}} f_{k} \frac{1}{\sqrt{M}} \sum_{m=0}^{M-1} d_{m} \mathrm{e}^{-\mathrm{j} 2 \pi k \frac{m}{M}} \mathrm{e}^{\mathrm{j} 2 \pi k \frac{n}{N}},
$$

where $f_{k}=\sqrt{M} c_{k} / \sqrt{\sum_{k=L_{\mathrm{d}}}^{L_{\mathrm{u}}}\left|c_{k}\right|^{2}}$ is the $k$ th normalized FDSS coefficien 1 and $d_{i_{\ell}}=\sqrt{E_{\mathrm{s}}} \times s_{\ell}$ for $\ell=0,1, \ldots, L-1$, otherwise 0 , and $E_{\mathrm{s}}=M / L$ is the symbol energy.

In Fig. 2, the transmitter and receiver block diagrams are given for CSC-IM. First, the modulation symbols are obtained based on $\boldsymbol{i}$ and $\boldsymbol{h}$. An $M$-point DFT of the modulation symbols is then calculated. After the resulting sequence is shaped in the frequency domain with an FDSS for generating CSCs, the shaped sequence is mapped to the OFDM subcarriers. The discrete-time signal in (8) is obtained by applying an $N$ point inverse discrete Fourier transform (DFT) (IDFT) to the mapped sequence and prepending a $\mathrm{CP}$ with the duration of $T_{\mathrm{CP}}=N_{\mathrm{CP}} T_{\text {sample }}$ to the signal, where $N_{\mathrm{CP}}$ is the number of samples in the CP duration. At the RXc, after removing the $\mathrm{CP}$ and applying an $\mathrm{N}$-point DFT to the received signal, the signal in the frequency domain can be expressed as

$$
b_{k}=\frac{\lambda_{k} f_{k}}{\sqrt{M}} \sum_{m=0}^{M-1} d_{m} \mathrm{e}^{-\mathrm{j} 2 \pi k \frac{m}{M}}+\eta_{k},
$$

\footnotetext{
${ }^{1}$ The normalization is applied as the coefficients are truncated in 1 .
}

where $\eta_{k}$ is zero-mean additive white Gaussian noise (AWGN) with the variance $\sigma_{\mathrm{n}}^{2}$ and $\lambda_{k}$ is the CFR between TX and RXc for the $k$ th subcarrier for $k \in\left\{L_{\mathrm{d}}, L_{\mathrm{d}}+1, \ldots, L_{\mathrm{u}}\right\}$. In this study, we consider LMMSE frequency-domain equalization (FDE) followed by an $M$-point IDFT to obtain the modulation symbols, which can be given by

$$
\tilde{d}_{l}=\frac{1}{\sqrt{M}} \sum_{k=L_{\mathrm{d}}}^{L_{\mathrm{u}}} \underbrace{\frac{\lambda_{k}^{*} f_{k}^{*}}{\left|\lambda_{k} f_{k}\right|^{2}+\sigma_{\mathrm{n}}^{2}} b_{k}}_{\text {LMMSE-FDE }} \mathrm{e}^{\mathrm{j} 2 \pi\left(k-L_{\mathrm{d}}\right) \frac{l}{M}},
$$

for $l \in\{1,2, \ldots, M-1\}$. Note that (10) explicitly shows that an equalizer needs to be employed even in AWGN channel (i.e., $\lambda_{k}=1$ ) for CSC-IM due to the FDSS coefficients for CSCs.

Without any constraint on the indices, the ML detector for $\boldsymbol{i}$ and $\boldsymbol{h}$ can be expressed as

$$
\{\hat{\boldsymbol{i}}, \hat{\boldsymbol{h}}\}=\arg \max _{\substack{i_{\ell} \in\{0, \ldots, M-1\} \\ i_{p}<\dot{i}_{r} \text { for } p<r \\ \dot{h}_{\ell} \in \mathbb{Z}_{H}}} \Re\left\{\sum_{\ell=0}^{L-1} \tilde{d}_{\dot{i}_{\ell}} \mathrm{e}^{-\mathrm{j} 2 \pi \dot{h}_{\ell} / H}\right\},
$$

where $\hat{\boldsymbol{i}} \triangleq\left(\hat{i}_{0}, \hat{i}_{1}, \ldots, \hat{i}_{L-1}\right)$ and $\hat{\boldsymbol{h}} \triangleq\left(\hat{h}_{0}, \hat{h}_{1}, \ldots, \hat{h}_{L-1}\right)$ are the detected chirp and the PSK symbol indices, respectively. Thus, a low-complexity ML detector can be implemented by evaluating each $\hat{d}_{l, z} \triangleq \Re\left\{\tilde{d}_{l} \mathrm{e}^{-\mathrm{j} 2 \pi z / H}\right\}$ for $l \in\{0,1, \ldots, M-$ $1\}$ and $z \in \mathbb{Z}_{H}$ and choosing $L$ indices and the corresponding $z$ values that maximize $\hat{d}_{l, z}$ [36].

Let $S_{q} \in \mathbb{Z}_{0}^{+}$denote the number of integers between two adjacent indices in a circular manner for $q \in\{1, \ldots, L\}$ as

$$
S_{q} \triangleq\left\{\begin{array}{ll}
i_{q}-i_{q-1}-1, & 1 \leq q<L \\
M-1-i_{L-1}+i_{0}, & q=L
\end{array} .\right.
$$

With the IS, we introduce constraints on the indices such that $S_{q} \geq \Delta$ for $q \in\{1, \ldots, L\}$ and $\Delta \in \mathbb{Z}_{0}^{+}$to improve the range estimation accuracy by ensuring a low AC zone in the case of simultaneous CSCs transmissions. A realization under IS for $\Delta=2$ and $M=10$ is given in Fig. 2, where $\left(i_{0}, i_{1}, i_{2}\right)=$ $(0,4,7)$ and $\left(S_{1}, S_{2}, S_{3}\right)=(3,2,2)$. We discuss the IS and its impact on TX and RXc in Section IV-A in detail.

\section{A. Theoretical error-rate analysis}

Consider the case where the FDSS is not utilized, i.e., DFT-s-OFDM-IM. Let $\mathrm{E}_{l, z \mid i, \boldsymbol{h}}$ denote the event where $\hat{d}_{l, z}$ is larger than or equal to at least one of the elements of $\left\{\hat{d}_{i_{\ell}, h_{\ell}}\right\}$. 
Hence, based on De Morgan's law, $P\left(\mathrm{E}_{l, z \mid i, \boldsymbol{h}}\right)$ can be obtained as

$$
\begin{aligned}
P\left(\mathrm{E}_{l, z \mid i, \boldsymbol{h}}\right) & =P\left(\bigcup_{\ell=0}^{L-1} \mathrm{E}_{l, z \mid i_{\ell}, h_{\ell}}\right)=1-P\left(\bigcap_{\ell=0}^{L-1} \mathrm{E}_{l, z \mid i_{\ell}, h_{\ell}}^{\mathrm{c}}\right) \\
& =1-\prod_{\ell=0}^{L-1}\left(1-P\left(\mathrm{E}_{l, z \mid i_{\ell}, h_{\ell}}\right)\right) .
\end{aligned}
$$

A block error occurs if $l$ is not an element of $\boldsymbol{i}$ or $z \neq h_{\ell}$ for $i_{\ell}=l$. The probability of block error can then be expressed as

$$
P_{\mathrm{e}}=P(\underbrace{\bigcup_{l=0}^{M-1} \bigcup_{z=0}^{H-1}}_{\substack{(l, z) \neq\left(i_{\ell}, h_{\ell}\right) \\ \ell \in\{0, \ldots L-1\}}} \mathrm{E}_{l, z \mid i, \boldsymbol{h}}) \leq \underbrace{\sum_{l=0}^{M-1} \sum_{z=0}^{H-1}}_{\substack{(l, z) \neq\left(i_{\ell}, h_{\ell}\right) \\ \ell \in\{0, \ldots L-1\}}} P\left(\mathrm{E}_{l, z \mid i, \boldsymbol{h}}\right) .
$$

By using (13), (14) can be given by

$$
\begin{aligned}
P_{\mathrm{e}} & \leq \underbrace{\sum_{l=0}^{M-1} \sum_{z=0}^{H-1}}_{\substack{(l, z) \neq\left(i_{\ell}, h_{\ell}\right) \\
\ell \in\{0, \ldots L-1\}}}\left(1-\prod_{\ell=0}^{L-1}\left(1-P\left(\mathrm{E}_{l, z \mid i_{\ell}, h_{\ell}}\right)\right)\right) \\
& =\underbrace{\sum_{l=0}^{M-1} \sum_{z=0}^{H-1}}\left(1-\prod_{\ell=0}^{L-1}\left(1-P\left(\mathrm{E}_{l, z \mid i_{\ell}, h_{\ell}}\right)\right)\right) \\
& +\sum_{\ell=0}^{L-1} \underbrace{H-1}_{z \neq h_{\ell}}\left(1-\prod_{\ell=0}^{L-1}\left(1-P\left(\mathrm{E}_{l, z \mid i_{\ell}, h_{\ell}}\right)\right)\right) .
\end{aligned}
$$

The Euclidean distance between $d_{i_{\ell}}$ and $d_{l \neq i_{\ell}}$ is fixed and can be calculated as $d_{\text {ind }}=\sqrt{2 E_{\mathrm{s}}}$. The minimum Euclidean distance between two PSK constellation points for the same index is $d_{\mathrm{psk}}=2 \sqrt{E_{\mathrm{s}}} \sin \left(\frac{\pi}{H}\right)$. Under the coherent detection and by using the symbol-error rate for $H$-PSK [32], this implies that the right-hand side of (15) can be re-written as

$$
\begin{aligned}
P_{\mathrm{e}} \leq U_{L} \triangleq(M-L) H( & \left.1-\left(1-Q\left(\frac{d_{\text {ind }}}{\sqrt{2 N_{0}}}\right)\right)^{L}\right) \\
& +L\left(1-\left(1-P_{H-\mathrm{PSK}}\right)^{L}\right),
\end{aligned}
$$

where

$$
P_{H \text {-PSK }}=\left\{\begin{array}{ll}
2 Q\left(\frac{d_{\mathrm{psk}}}{\sqrt{2 N_{0}}}\right), & H \geq 4 \\
Q\left(\frac{d_{\mathrm{psk}}}{\sqrt{2 N_{0}}}\right), & H=2 \\
0, & H=1
\end{array},\right.
$$

$Q(\cdot)$ is the Q-function, and $U_{L}$ is the UB of the probability of error for any $L \in\{1, \ldots, M\}$ and $H \geq 1$ for DFT-s-OFDM-IM in AWGN channel.

Now consider the case where the FDSS for CSCs is included, i.e., CSC-IM. Since we use a single-tap LMMSEFDE followed by an $M$-point IDFT as in (10), inter-symbol

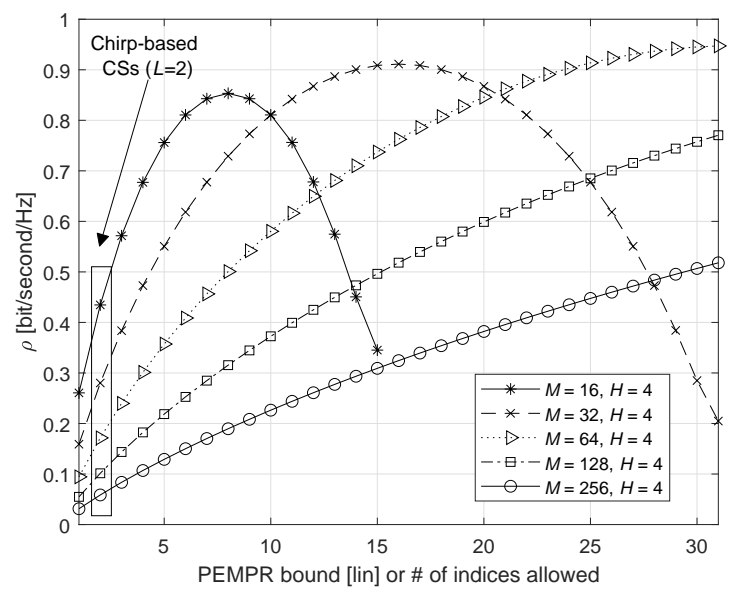

Fig. 3. Trade-off between the SE and the maximum PMEPR for CSC-IM

interfence (ISI) occurs and the noise on the received modulation symbols becomes correlated. By utilizing the derivation in [37] for a typical DFT-s-OFDM transmission with independent and identically distributed (i.i.d.) modulation symbols under a fading channel, the signal-to-noise ratio (SNR) for the received modulation symbols after the equalization can be obtained as $S N R_{\text {post }}=1 /\left(\sqrt{1 / \alpha_{\mathrm{MMSE}}}-1\right)$, where $\alpha_{\mathrm{MMSE}}=\left(1 / M \sum_{k=L_{\mathrm{d}}}^{L_{\mathrm{u}}}\left|f_{k}\right|^{2} /\left(\left|f_{k}\right|^{2}+\sigma_{\mathrm{n}}^{2}\right)\right)^{2}$. Assuming that the noise on the modulation symbols is uncorrelated and follows Gaussian distribution, the bound in (16) can be calculated by using $N_{0}=1 / S N R_{\text {post }}$. It is worth noting that these assumptions are weak as long as the parameters do not cause ill-conditioned operations (e.g., $D \ll M$ ) and (16) is fairly accurate for typical FDSS choices, e.g., (4), as shown in Section $\mathrm{V}$

\section{B. Trade-off between PMEPR and Spectral Efficiency}

The CSC-IM allows $p=p_{1}+p_{2}$ information bits to be transmitted, where $p_{1}=\left\lfloor\log _{2}\left(\left(\begin{array}{c}M \\ L\end{array}\right)\right)\right\rfloor$ and $p_{2}=L \log _{2}(H)$ since $L$ indices can be chosen from $M$ indices in $\left(\begin{array}{c}M \\ L\end{array}\right)$ different ways and $L H$-PSK symbols are utilized. Hence, the SE of the scheme can be calculated as $\rho=\left\lfloor\log _{2}\left(\left(\begin{array}{c}M \\ L\end{array}\right) \times H^{L}\right)\right\rfloor / M$ $\mathrm{bit} / \mathrm{second} / \mathrm{Hz}$. Therefore, the SE increases with $L \leq\lfloor M / 2\rfloor$. On the other hand, since chirps are constant-envelope signals, i.e., $\left|B_{l}(t)\right|=1$, the maximum amplitude of the superposition of $L$ chirps is less than or equal to $L$, i.e., the instantaneous power is maximum $L^{2}$. As the mean power is $L$ in the presence of random PSK symbols, the PMEPR of the signal with CSC-IM is always less than or equal to $L$. Therefore, the proposed scheme leads to a trade-off between maximum PMEPR and SE as quantified in Fig. 3 by sweeping $L$ for a given $L$ and for a quadrature PSK (QPSK) constellation, i.e., $H=4$, and $M \in\{16,32,64,128,256\}$. While the SE of CSC-IM increases with a larger $L \leq\lfloor M / 2\rfloor$, the maximum PMEPR is always less than or equal to $10 \log _{10} L \mathrm{~dB}$. This trade-off can be helpful to identify the maximum number of CSCs and the corresponding SE for a given power back-off. For example, if the tolerable input power back-off is $7 \mathrm{~dB}$, the 
maximum number of active CSCs is 5 and the maximum SE is 0.35 bits/(s.Hz) for $M=64$ based on Fig. 3

As a special case, the proposed scheme for $L=2$ reveals that non-trivial CSs can be generated from chirps as follows:

Theorem 1. Let $x(t)$ and $y(t)$ be the signals given by

$$
\begin{aligned}
& x(t)=d_{p} \mathrm{e}^{\mathrm{j} \psi_{p}(t)}+d_{r} \mathrm{e}^{\mathrm{j} \psi_{r}(t)}, \\
& y(t)=d_{p} \mathrm{e}^{\mathrm{j} \psi_{p}(t)}-d_{r} \mathrm{e}^{\mathrm{j} \psi_{r}(t)},
\end{aligned}
$$

for $d_{p}, d_{r} \in \mathbb{C}$ and $\left|d_{p}\right|=\left|d_{r}\right|=1$. The Fourier coefficients of $x(t)$ and $y(t)$ form a GCP.

Proof. By the definition of a GCP, we need to show that $|x(t)|^{2}+|y(t)|^{2}$ is constant:

$$
\begin{aligned}
|x(t)|^{2}= & \left|d_{p}\right|^{2}+\left|d_{r}\right|^{2} \\
& +d_{p} d_{r}^{*} \mathrm{e}^{\mathrm{j}\left(\psi_{p}(t)-\psi_{r}(t)\right)}+d_{p}^{*} d_{r} \mathrm{e}^{-\mathrm{j}\left(\psi_{p}(t)-\psi_{r}(t)\right)} .
\end{aligned}
$$

Similarly,

$$
\begin{aligned}
|y(t)|^{2}= & \left|d_{p}\right|^{2}+\left|d_{r}\right|^{2} \\
& -d_{p} d_{r}^{*} \mathrm{e}^{\mathrm{j}\left(\psi_{p}(t)-\psi_{r}(t)\right)}-d_{p}^{*} d_{r} \mathrm{e}^{-\mathrm{j}\left(\psi_{p}(t)-\psi_{r}(t)\right)} .
\end{aligned}
$$

Therefore, $|x(t)|^{2}+|y(t)|^{2}=2 \times\left(\left|d_{p}\right|^{2}+\left|d_{r}\right|^{2}\right)=4$, which implies that $\mathcal{F}\{x(t)\}$ and $\mathcal{F}\{y(t)\}$ form a GCP.

Theorem 1 indicates that the Fourier coefficients of a linear combination of the frequency responses of two constantenvelope chirps result in a CS. Hence, based on (4) and (5), Fresnel integrals and Bessel functions can be useful for generating CSs, which have not been reported in the literature to the best of our knowledge.

Example 1. Assume that $x(t)$ and $y(t)$ are linear combinations of two circularly-shifted versions of a band-limited sinusoidal chirp. By using (1) and (5), the Fourier coefficients of $x(t)$ and $y(t)$ are obtained as

$$
\begin{aligned}
& a_{k}=d_{p} J_{k}\left(\frac{D}{2}\right) \mathrm{e}^{-\mathrm{j} 2 \pi k \frac{\tau_{p}}{T_{\mathrm{s}}}}+d_{r} J_{k}\left(\frac{D}{2}\right) \mathrm{e}^{-\mathrm{j} 2 \pi k \frac{\tau_{r}}{T_{\mathrm{s}}}}, \\
& b_{k}=d_{p} J_{k}\left(\frac{D}{2}\right) \mathrm{e}^{-\mathrm{j} 2 \pi k \frac{\tau_{p}}{T_{\mathrm{s}}}}-d_{r} J_{k}\left(\frac{D}{2}\right) \mathrm{e}^{-\mathrm{j} 2 \pi k \frac{\tau_{r}}{T_{\mathrm{s}}}}
\end{aligned}
$$

respectively. Based on Theorem [1 $\left(a_{i}\right)_{i=-\infty}^{\infty}$ and $\left(b_{i}\right)_{i=-\infty}^{\infty}$ form a GCP. Since the sinusoidal chirps are band-limited signals, the amplitude of a Fourier coefficient approaches to zero for $|i| \geq D / 2$. Therefore, $\left(a_{i}\right)_{i=L_{\mathrm{d}}}^{L_{\mathrm{u}}}$ and $\left(b_{i}\right)_{i=L_{\mathrm{d}}}^{L_{\mathrm{u}}}$ are approximately GCP. Note that if the sinusoidal chirps are replaced by the linear chirps, by using (4), the Fourier coefficients of $x(t)$ and $y(t)$ can be calculated as

$$
\begin{aligned}
a_{k}= & d_{p} \gamma_{k}\left(C\left(\alpha_{k}\right)+C\left(\beta_{k}\right)+\mathrm{j} S\left(\alpha_{k}\right)+\mathrm{j} S\left(\beta_{k}\right)\right) \mathrm{e}^{-\mathrm{j} 2 \pi k \frac{\tau_{p}}{T_{\mathrm{s}}}} \\
& +d_{r} \gamma_{k}\left(C\left(\alpha_{k}\right)+C\left(\beta_{k}\right)+\mathrm{j} S\left(\alpha_{k}\right)+\mathrm{j} S\left(\beta_{k}\right)\right) \mathrm{e}^{-\mathrm{j} 2 \pi k \frac{\tau_{r}}{T_{\mathrm{s}}}}, \\
b_{k}= & d_{p} \gamma_{k}\left(C\left(\alpha_{k}\right)+C\left(\beta_{k}\right)+\mathrm{j} S\left(\alpha_{k}\right)+\mathrm{j} S\left(\beta_{k}\right)\right) \mathrm{e}^{-\mathrm{j} 2 \pi k \frac{\tau_{p}}{T_{\mathrm{s}}}} \\
& -d_{r} \gamma_{k}\left(C\left(\alpha_{k}\right)+C\left(\beta_{k}\right)+\mathrm{j} S\left(\alpha_{k}\right)+\mathrm{j} S\left(\beta_{k}\right)\right) \mathrm{e}^{-\mathrm{j} 2 \pi k \frac{\tau_{r}}{T_{\mathrm{s}}}} .
\end{aligned}
$$

In Fig. 4, we exemplify a GCP of length $M=24$, synthesized through (20) and (21) for $L_{\mathrm{d}}=-11, L_{\mathrm{u}}=12$, $\tau_{p} / T_{\mathrm{s}}=0 / 24, \tau_{r} / T_{\mathrm{s}}=1 / 24$, and $d_{p}=d_{r}=1$. When $D=24$, the sequences are truncated heavily. Therefore, it does not satisfy the definition of a GCP given in Section II-B.
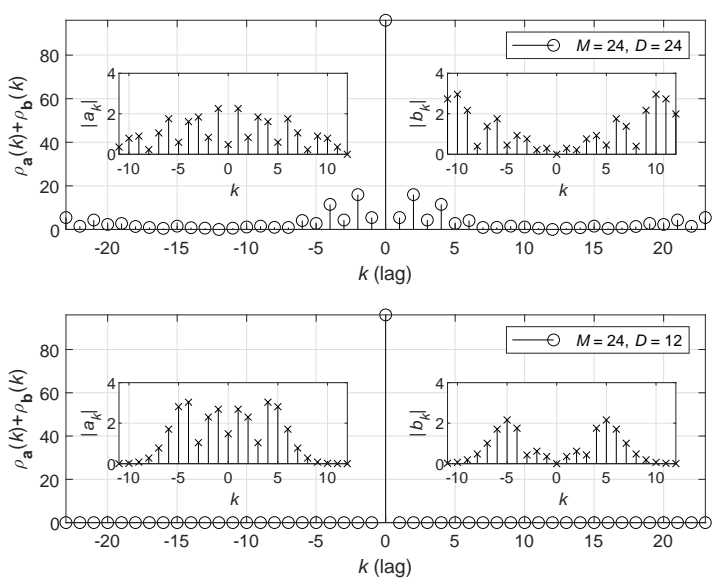

Fig. 4. A GCP of length $M=24$ synthesized with sinusoidal chirps and the impact of truncation on the GCP formation.

On the other hand, when the maximum frequency deviation is halved, $M_{\mathrm{ocb}}$ is 15 for containing $99 \%$ of the total integrated power of the spectrum. Hence, $M=24$ forms the chirps well and the resulting sequences form a GCP. It is also worth noting that synthesized CSs are not unimodular sequences. Therefore, the mean power of OFDM symbol changes although instantaneous power is bounded.

Corollary 1. Let $\mathbb{W}=\left\{B_{m}(t) \mid m=0,1, \ldots, M-1\right\}$ be a set of $M C S C s$ of an arbitrary band-limited function with the duration $T_{\mathrm{s}}$ and $M_{\mathrm{ocb}} \leq M$. Without using the same CSC twice, the total number of distinct CSs of length $M$ is $\left(\begin{array}{c}M \\ 2\end{array}\right) \times H^{2}$ for $d_{p}, d_{r} \in\left\{\mathrm{e}^{\mathrm{j} 2 \pi z / H} \mid z=0,1, \ldots, H-1\right\}$.

Proof. There exist $H^{2}$ combinations for $\left\{d_{p}, d_{r}\right\}$ and $\mathrm{e}^{\mathrm{j} \psi_{p}(t)}$ and $\mathrm{e}^{\mathrm{j} \psi_{r}(t)}$ in (18) can be chosen in $\left(\begin{array}{c}M \\ 2\end{array}\right)$ ways without using the same chirp. Thus, the total number of CSs is $\left(\begin{array}{c}M \\ 2\end{array}\right) \times H^{2}$ via Theorem 1 . The CSs are distinct as $B_{m}(t)$ are distinct for $m \in\{0,1, \ldots, M-1\}$. Since the OCB of $B_{m}(t)$ is less than or equal to $M / T_{\mathrm{s}}$, the length of the synthesized CS is $M$ based on Nyquist's sampling theorem.

Note that the extension of Theorem 1 for $L>2$ is currently a difficult open problem. To address this issue, using the coefficients of CSCs as seed GCP sequences for the recursive $\mathrm{CS}$ construction [12] is a potential direction that can be pursued.

\section{Practical Issues}

At the transmitter, mapping the information bits to a combination of $L$ indices (and vice versa for RXr) may be a challenge. This can be addressed by constructing a bijective function from integers to the set of combinations via a combinatorial number system of degree $L$ [27], also called combinadics. Note that we investigate a generalization of combinadics in Section IV-A2 to obtain a mapping rule under the proposed IS to develop the encoder and decoder, which may also be used for the unconstrained case.

The choice of FDSS is important for obtaining a low error rate and a low PMEPR. In [10], it was demonstrated that a 
flatter FDSS improves the bit error rate (BER) performance for the receiver with a single-tap LMMSE-FDE. For example, a linear chirp offers a more flat FDSS as compared to the one with a sinusoidal chirp. On the other hand, a linear chirp causes abrupt instantaneous frequency changes within the IDFT duration. Therefore, it requires a much lower $D$ as compared to the one for a sinusoidal chirp for a given $M$ to form a CS. This issue can distort the signals and cause a larger PMEPR than the theoretical bound, as demonstrated in Section V. Note that the PMEPR can be reduced to the theoretical limit if FDSS is allowed to be extended to the sidebands with some roll-off factor, i.e., a larger $L_{\mathrm{u}}$ and a lower $L_{\mathrm{d}}$ in (3).

\section{Comparisons}

As compared to DFT-s-OFDM-IM, the proposed scheme has a significant PMEPR advantage since the signal is spread in the time domain whereas DFT-s-OFDM generates Dirichlet sinc pulses. The proposed scheme and OFDM-IM have similar PMEPR characteristics since OFDM-IM also spreads the symbol energy in time 2 . However, the energy is also distributed within the signal bandwidth with the proposed scheme. Thus, the proposed scheme allows a coherent receiver to exploit frequency diversity in frequency-selective channels naturally. On the other hand, OFDM-IM receiver does not fully benefit from the frequency selectivity without an extra operation, e.g., repetitions or interleaving [38], at the transmitter.

In [11], a low PMEPR coding scheme was proposed to generate $H$-PSK CSs-RM. This scheme synthesizes $H^{m+1} \times m ! / 2$ CSs, where the length of each CS must be in the form of $2^{m}$, where $m \in \mathbb{Z}^{+}$. When a seed GCP of length $N$ is utilized with this scheme, it can be shown that $H^{m+1} \times m$ ! CSs of length $N \cdot 2^{m}$ can be generated [12]. Therefore, the spectral efficiency of the schemes in [11] and [12] can be calculated as $\left\lfloor\log _{2}\left(H^{m+1} \times m ! / 2\right)\right\rfloor / 2^{m}$ and $\left\lfloor\log _{2}\left(H^{m+1} \times m !\right)\right\rfloor /\left(N 2^{m}\right)$ $\mathrm{bit} / \mathrm{second} / \mathrm{Hz}$, respectively. The differences between these schemes and the proposed scheme can be listed as follows: 1) The proposed scheme supports flexible bandwidth. For example, $M$ can be an integer chosen as an integer multiple of 12 based on the resource allocation in 3GPP 5G NR and 4G LTE. 2) The schemes in [11], [12] do not provide a tradeoff between PMEPR and spectral efficiency whereas $L$ can be chosen for a higher SE at a cost of high PMEPR with our scheme. The PMEPR is still theoretically limited. 3) The number of CSs is a function of a second-order coset term generated through permutations in [11] and [12]. However, designing a decoder for all possible permutations is not trivial [12], [39]. For a fixed coset, the decoder can be implemented through fast Hadamard transformation or recursive methods [40], but the SE reduces to $\left\lfloor\log _{2} H^{m+1}\right\rfloor$. Under this case, the SE of the proposed scheme and the schemes in [11] and [12] are similar while a simple decoder can be employed for the proposed method.

Note that the SE of the proposed scheme is low as compared to typical coding schemes such as low-density parity-

\footnotetext{
${ }^{2}$ In this study, we consider a large number of subcarriers with few indices for both OFDM-IM and DFT-s-OFDM-IM.
}

check (LDPC) or polar codes. Although this appears as a disadvantage, there exist many communication scenarios (e.g., uplink control channels in 5G NR [41], Internet-of-Things (IoT) networks) where the primary concern is reliability under low SNR or a longer battery life, rather than a higher data rate. In addition, to exploit the demodulated data for improving radar functionality for bi-static DFRC scenarios, the signal should be able to decoded at very low SNR. For these scenarios, the proposed scheme provides a way of limiting PMEPR without an optimization procedure at the transmitter while exploiting frequency selectivity and supporting radar functionality discussed next.

\section{RADAR FUnCTIONALITY WITH CSC-IM}

At the RXr, similar to (9), the received signal in the frequency domain can be expressed as

$$
b_{k}=\frac{\zeta_{k} f_{k}}{\sqrt{M}} \sum_{m=0}^{M-1} d_{m} \mathrm{e}^{-\mathrm{j} 2 \pi k \frac{m}{M}}+\eta_{k}
$$

where $\eta_{k}$ is zero-mean AWGN with the variance of $\sigma_{\mathrm{n}}^{2}$. The received symbols in (22) can be re-expressed in the vector form as

$$
\mathbf{b}=\underbrace{\operatorname{diag}\{\mathbf{f}\} \operatorname{diag}\left\{\mathbf{D}_{M} \mathbf{d}\right\}}_{\mathbf{W} \triangleq \operatorname{diag}\{\mathbf{w}\}} \mathbf{h}+\mathbf{n},
$$

where $\mathbf{b}=\left[b_{L_{\mathrm{d}}}, \ldots, b_{L_{\mathrm{u}}}\right]^{\mathrm{T}}, \mathbf{f}=\left[f_{L_{\mathrm{d}}}, \ldots, f_{L_{\mathrm{u}}}\right], \mathbf{D}_{M}$ is the $M$-point normalized DFT matrix, $\mathbf{d}=\left[d_{L_{\mathrm{d}}}, \ldots, d_{L_{\mathrm{u}}}\right]^{\mathrm{T}}, \mathbf{n}=$ $\left[\eta_{L_{\mathrm{d}}}, \eta_{L_{\mathrm{d}}+1}, \ldots, \eta_{L_{\mathrm{u}}}\right]^{\mathrm{T}}, \mathbf{w}=\left[w_{L_{\mathrm{d}}}, \ldots, w_{L_{\mathrm{u}}}\right]^{\mathrm{T}}$ is the response of the waveform in the frequency, and $\mathbf{h}=\left[\zeta_{L_{\mathrm{d}}}, \ldots, \zeta_{L_{\mathrm{u}}}\right]^{\mathrm{T}}$. Based on (7), $\mathbf{h}$ can be expressed as

$$
\mathbf{h}=\mathbf{T a},
$$

where $\mathbf{T}=\left[\begin{array}{llll}\mathbf{t}_{\tau_{1}} & \mathbf{t}_{\tau_{2}} & \cdots & \mathbf{t}_{\tau_{R}}\end{array}\right] \in \mathbb{C}^{M \times R}$ is the delay matrix and $\mathbf{t}_{\tau_{s}}=\mathrm{e}^{-\mathrm{j} 2 \pi f_{\mathrm{c}} \tau_{s}} \times\left[\mathrm{e}^{-\mathrm{j} 2 \pi L_{\mathrm{d}} \frac{\tau_{s}}{T_{\mathrm{s}}}}, \cdots, \mathrm{e}^{-\mathrm{j} 2 \pi L_{\mathrm{u}} \frac{\tau_{s}}{T_{\mathrm{s}}}}\right]^{\mathrm{T}}$, and $\mathbf{a}=$ $\left[\alpha_{1}, \alpha_{2}, \ldots, \alpha_{R}\right]^{\mathrm{T}}$. For our DFRC scenario, the sequences $\boldsymbol{s}$ and $i$ are available at the RXr. Therefore, the symbols on the subcarriers, i.e., w, can be used as reference symbols. Hence, in AWGN channel, the ML-based delay estimation problem can be expressed as

$$
\begin{aligned}
\left\{\left(\tilde{\tau}_{s}, \tilde{\alpha}_{s}\right)\right\} & =\arg \min _{\substack{\left\{\left(\dot{\tau}_{s}, \dot{\alpha}_{s}\right)\right\} \\
s=1, \ldots, R}}\|\mathbf{b}-\mathbf{W} \dot{\mathbf{T}} \dot{\mathbf{a}}\|_{2}^{2} \\
& =\arg \min _{\substack{\left\{\left(\dot{\tau}_{s}, \dot{\alpha}_{s}\right)\right\} \\
s=1, \ldots, R}}\|\mathbf{W} \dot{\mathbf{T}} \dot{\mathbf{a}}\|_{2}^{2}-2 \Re\left\{\dot{\mathbf{a}}^{\mathrm{H}} \dot{\mathbf{T}}^{\mathrm{H}} \mathbf{W}^{\mathrm{H}} \mathbf{b}\right\} .
\end{aligned}
$$

For a single target, i.e., $R=1$, 25 can be reduced to

$$
\tilde{\tau}_{1}=\arg \max _{\dot{\tau}_{1}}\left|\Re\left\{\mathbf{t}_{\dot{\tau}_{1}}{ }^{\mathrm{H}} \mathbf{W}^{\mathrm{H}} \mathbf{b}\right\}\right|
$$

where $\tilde{\alpha}_{1}=\Re\left\{\mathbf{t}_{\tilde{\tau}_{1}}{ }^{\mathrm{H}} \mathbf{W}^{\mathrm{H}} \mathbf{b}\right\} /\left(\mathbf{w}^{\mathrm{H}} \mathbf{w}\right)$ by equating the derivative of cost function with respect to $\dot{\tau}_{1}$ and $\dot{\alpha}_{1}$ to zeros. The reason for the absolute value in (26) is that $\alpha_{1}$ can be negative or positive. The solution of (26) corresponds to the optimum MF and the objective function can be evaluated via a computer search. Note that $\mathbf{t}_{\tilde{\tau}_{s}}$ is a function of the carrier frequency. Thus, the search should consider narrow enough steps to obtain 
the maximum. In this study, we utilize a refinement procure that increases the number of points around the coarse estimate point through chirp Z-transformation.

The solution of (25) is not trivial for $R>1$ and a bruteforce search can cause a high-complexity RXr. To address this issue, we utilize (26) and propose an iterative procedure by subtracting the information related to $(n-1)$ th target from the signal as

$$
\mathbf{b}^{(n)}=\mathbf{b}^{(n-1)}-\tilde{\alpha}_{n-1} \mathbf{W} \mathbf{t}_{\tilde{\tau}_{n-1}}
$$

for $\mathbf{b}^{(1)}=\mathbf{b}$. To increase accuracy further, after $\tilde{\tau}_{s}$ and $\tilde{\alpha}_{s}$ are estimated through iterations for $s=1, \ldots, R$, we re-use the estimates obtained from (27) and update $\tilde{\tau}_{n}$ and $\tilde{\alpha}_{n}$ by using

$$
\mathbf{b}^{(n)}=\mathbf{b}-\sum_{\substack{s=1 \\ s \neq n}}^{R} \tilde{\alpha}_{s} \mathbf{W} \mathbf{t}_{\tilde{\tau}_{s}}
$$

in (26). The corresponding range for $s$ th target can then be obtained as $\tilde{r}_{s}=\tilde{\tau}_{s} \times c / 2$. Based on our simulation trials, updating the estimates through (28) twice addresses the rootmean-square error (RMSE) floor in our previous results in [1].

The successful cancellation of the $(n-1)$ th reflected signal in (27) relies on the accurate estimate of the reflection coefficient. However, when there are multiple targets, the reflection coefficient estimation can be inaccurate due to 1) the distance between the targets and 2) the correlation properties of the waveform. The reason for the former issue is that multiple targets appear as a single target if the distance between two targets is less than the minimum resolution. It is wellknown that the minimum resolution can be calculated as $r_{\min }=0.5 \times c / B$ meters, where $B=D / T_{\mathrm{s}}$ is the chirp bandwidth. The issue related to the waveform can be seen in (26). For multiple CSCs transmission, the MF output, i.e., $\Re\left\{\mathbf{t}_{\tilde{\tau}_{1}}{ }^{\mathrm{H}} \mathbf{W}^{\mathrm{H}} \mathbf{b}\right\}$ for $\tilde{\tau}_{1} \in\left[0, T_{\mathrm{CP}}\right)$, is a superposition of the MF outputs of all CSCs and CSCs that are closer to each other in time can cause inaccurate estimations of the reflection coefficients. Also, the estimation accuracy for path delays can degrade since the reward function in (26) can be high at different values of $\dot{\tau}_{1}$ for $L>1$, i.e., multiple spikes, although there is a single target, To address the correlation problem, we investigate two solutions: 1) IS unique to the proposed scheme and 2) Range estimation over the LMMSE channel estimate, i.e., removing the impact of waveform on the range estimation.

\section{A. Solution \#1: Index Separation for MF-based estimation}

For this solution, we consider the MF-based estimation and use the iterations in 27) at the RXr. However, we modify the transmitter such that the spikes in the AC function occurring due to the multiple CSC transmissions are well-separated. To this end, we restrict the selected CSC indices as the distance between two adjacent indices is larger than a certain value, which separates CSCs apart in time as in Fig. 2. This restriction improves the accuracy of the reflection coefficient estimation in (26) and the accuracy of the cancellations in 27. by guaranteeing a low AC zone.
IS introduces a trade-off between communications and radar. This is because a larger $\Delta$ for improving the radar functionality can degrade the SE of the proposed scheme. Hence, the first question that we need to address is how the SE of CSC-IM is affected for a given $\Delta$. In addition, the restriction on indices under IS requires a new bijective mapping between bits and indices for the encoder and decoder designs.

1) Spectral Efficiency under IS: Let $\mathcal{A}_{L, \Delta}(M)$ denote the cardinality of the set of sequences $\left(i_{0}, i_{1}, \ldots, i_{L-1}\right)$, where $0 \leq$ $i_{p}<i_{r}<M$ for $p<r$ and $S_{q} \geq \Delta$ for all $q \in\{1,2, \ldots, L\}$, i.e., the number of valid index permutations for given $\Delta, L$ and $M$. Also, let $\mathcal{B}_{L, \Delta}(Z)$ denote the cardinality of the sequences $\left(S_{1}, S_{2}, \cdots, S_{L}\right)$ such that $S_{1}+S_{2}+\cdots+S_{L}=Z \in \mathbb{Z}_{0}^{+}$and $S_{q} \geq \Delta \in \mathbb{Z}_{0}^{+}$for $q \in\{1,2, \ldots, L\}$. To obtain $\mathcal{A}_{L, \Delta}(M)$, we need the following lemma:

Lemma 1. For $L \geq 1$,

$$
\begin{aligned}
\mathcal{B}_{L, \Delta}(Z) & =\sum_{r=\Delta}^{Z-\Delta L+\Delta} \mathcal{B}_{L-1, \Delta}(Z-r) \\
& =\left\{\begin{array}{ll}
\left(\begin{array}{cc}
Z-L \Delta+L-1 \\
L-1
\end{array}\right), & Z \geq \Delta L \\
0 . & \text { otherwise }
\end{array} .\right.
\end{aligned}
$$

Proof. The cardinality of the set of $\left(S_{1}, S_{2}, \cdots, S_{L-1}\right)$ is $\mathcal{B}_{L-1, \Delta}\left(Z-S_{L}\right)$ as $S_{1}+S_{2}+\cdots+S_{L-1}=Z-S_{L}$ for $S_{L} \in\{\Delta, \ldots, Z-\Delta L+\Delta\}$, which implies the recursive formula in 29.

It is well-known that the number of compositions of $n$ into exactly $k$ parts is $\left(\begin{array}{l}n-1 \\ k-1\end{array}\right)$, where each part is greater than 0 .We define the variable $S_{q}^{\prime}$ by setting $S_{q}^{\prime}=S_{q}-(\Delta-1)$.

- Case $1(\Delta \geq 1): S_{1}^{\prime}+S_{2}^{\prime}+\cdots+S_{L}^{\prime}=Z-L(\Delta-1)$ holds. Hence, $\mathcal{B}_{L, \Delta}(Z)$ must be equal to the number of compositions of $Z-L(\Delta-1)$ into exactly $L$ parts, which implies (30) for $\Delta>1$.

- Case $2(\Delta=0)$ : Since $S_{1}^{\prime}+S_{2}^{\prime}+\cdots+S_{L}^{\prime}=Z+L$ holds, $\mathcal{B}_{L, 1}(Z)$ must be equal to $\left(\begin{array}{c}Z+L-1 \\ L-1\end{array}\right)$.

If $Z<\Delta L$, there exists no composition.

Theorem 2. For $L \geq 2$,

$$
\mathcal{A}_{L, \Delta}(M)=\left\{\begin{array}{ll}
\frac{M}{L}\left(\begin{array}{c}
M-L \Delta-1 \\
L-1
\end{array}\right), & M \geq L(\Delta+1) \\
0, & \text { otherwise }
\end{array} .\right.
$$

Proof. Consider the following steps:

Step 1: By the definition in (12), the cardinality of the set of sequences $\left(i_{0}, S_{1}, \ldots, S_{L-1}\right)$ is $\mathcal{A}_{L, \Delta}(M)$ and $S_{1}+S_{2}+$ $\cdots+S_{L}=M-L$. Since $S_{q} \geq \Delta$ for all $q$, the inequality given by

$$
\Delta \leq S_{L} \leq U
$$

holds for $U \triangleq M-L(\Delta+1)+\Delta$.

Step 2: By the definition in (12), $S_{L}$ can be expressed as $S_{L}=i_{0}+b$, where $b=M-1-i_{L-1}$. Therefore, for $i_{0} \in$ $\{0,1, \ldots, U\}$, the inequality in (32) can be re-stated as

$$
\Delta-i_{0} \leq b \leq U-i_{0} .
$$

Step 3: Since $S_{q} \geq \Delta$ for all $q$ and $S_{1}+S_{2}+\cdots+S_{L}=$ $M-L$, the cardinality of the sequences $\left(S_{1}, S_{2}, \cdots, S_{L-1}\right)$ 


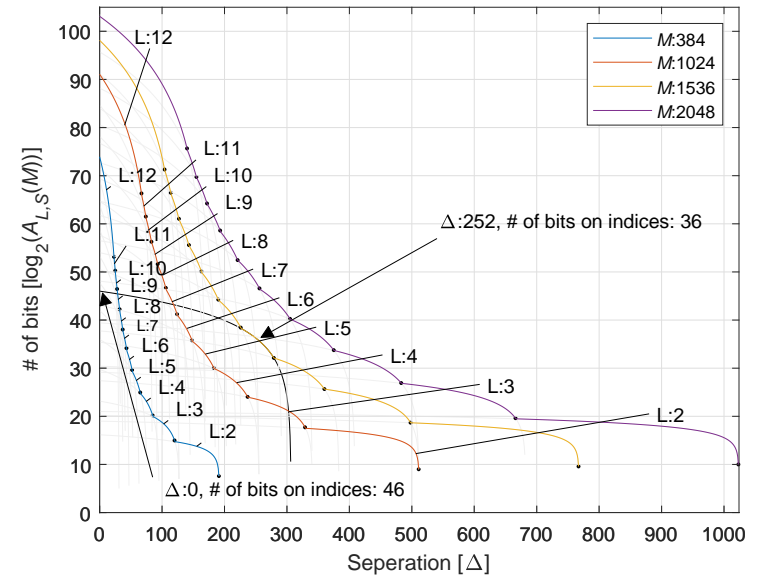

(a) Trade-off between separation and the number of information bits on the indices.

Fig. 5. The impact of IS on the SE.

is $\mathcal{B}_{L-1, \Delta}\left(M-L-S_{L}\right)$ for a given $S_{L}$. Hence, by using (33) and Lemma 2. $\mathcal{A}_{L, \Delta}(M)$ can be expressed as

$$
\begin{aligned}
\mathcal{A}_{L, \Delta}(M) & =\sum_{i_{0}=0}^{U} \sum_{b=\max \left(0, \Delta-i_{0}\right)}^{U-i_{0}} \mathcal{B}_{L-1, \Delta}\left(M-L-\left(i_{0}+b\right)\right) \\
= & \sum_{i_{0}=0}^{\Delta-1} \mathcal{B}_{L, \Delta}(M-L)+\sum_{i_{0}=\Delta}^{U} \mathcal{B}_{L, \Delta}\left(M-L+\Delta-i_{0}\right) \\
= & \Delta \mathcal{B}_{L, \Delta}(M-L)+\mathcal{B}_{L+1, \Delta}(M-L+\Delta) \\
= & \frac{M}{L}\left(\begin{array}{c}
M-L \Delta-1 \\
L-1
\end{array}\right) .
\end{aligned}
$$

There exists no valid sequence for $M<L(\Delta+1)$, i.e., $\mathcal{A}_{L, \Delta}(M)=0$.

For a given $\Delta$, the SE of the CSC-IM can now be calculated as $\rho=\left\lfloor\log _{2}\left(\mathcal{A}_{L, \Delta}(M) \times H^{L}\right)\right\rfloor / M$. In Fig. [5] trade-off between $\Delta$ and the maximum number of information bits that are encoded with the indices, i.e., $\log _{2}\left(\mathcal{A}_{L, \Delta}(M)\right)$. As expected, a larger $\Delta$ means a lower number of information bits that can be transmitted on the indices. The degradation is more rapid with a larger $L$ although the number of information bits is larger for smaller values of $\Delta$.

One interesting question is that what is the largest $\Delta$ such that the SE still remains at the maximum for a given $L$ ? To address this question, let $\Delta_{\text {no-loss }}$ denote the largest separation such that $\left\lfloor\log _{2}\left(\begin{array}{c}M \\ L\end{array}\right)\right\rfloor=\left\lfloor\log _{2} \mathcal{A}_{L, \Delta_{\text {no-loss }}}(M)\right\rfloor$. Hence, $\mathcal{A}_{L, \Delta_{\text {no-loss }}}(M) \geq 2^{\left.\log _{2}\left(\begin{array}{c}M \\ L\end{array}\right)\right\rfloor}$ must hold. Since Theorem 2 provides a closed-form solution, $\Delta_{\text {no-loss }}$ can be evaluated with a computer search.

In Fig. [5] we we plot $\Delta_{\text {no-loss }}$ for a given $M$. The surprising result is that the separation between two adjacent indices can be as large as $M / 4-1$ without losing SE for $L=2$. For instance, for $M=2^{k}$, where $k \in \mathbb{Z}^{+}, \Delta_{\text {no-loss }}$ reaches its maximum value, i.e., $\Delta_{\text {no-loss }}=M / 4-1$. In other words, the duration of the low $\mathrm{AC}$ zone can be as large as a typical $\mathrm{CP}$ size as $\Delta_{\text {no-loss }} / M=N_{\mathrm{CP}} / N$ can be maintained. The value of

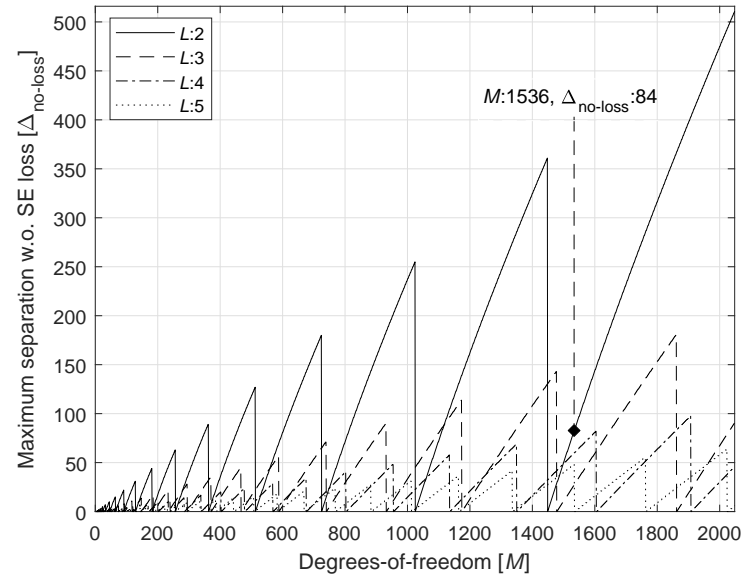

(b) $\Delta_{\text {no-loss }}$ versus $M$. The distance between two indices can be as large as $M / 4$ without losing $\mathrm{SE}$ for $L=2$.

$M$ where $\Delta_{\text {no-loss }}$ reaches at its maximum depends on $L$. For instance, the values of $M$ are 931, 954, and 1012 for $L=3$, $L=4$, and $L=5$, respectively, and the corresponding values for $\Delta_{\text {no-loss }}$ are 90,48 , and 31 . The ratios between $\Delta_{\text {no-loss }}$ and $M$ approach to $1 / 10.25,1 / 19.4$, and $1 / 32.45$ for $L=3, L=$ 4 , and $L=5$, respectively. We also observe abrupt changes in $\Delta_{\text {no-loss }}$ for different values of $M$. For example, $\Delta_{\text {no-loss }}$ becomes minimum, i.e., $\Delta_{\text {no-loss }}=0$, for $M=2^{k}+1$ for $M=2$. This behavior is because the number of bits encoded on the indices increases by 1 when $M$ increases by 1 .

2) Bijective mappings between natural numbers and indices under IS: To develop an encoder and a decoder for CSC-IM by taking the IS into account, information bits need to be mapped to the indices under a separation constraint or vice versa. We address this issue by deriving the mapping rules from a positive integer $n$ to the indices or vice versa for a given $\Delta$, where the information bits can be converted to $n$ through a binary to decimal conversion. The following definitions are needed:

Definition 1. The function $\epsilon_{\mathcal{A}}(n, M, L, \Delta)$ maps a positive integer $n$ to the sequence $\left(i_{0}, i_{1}, \ldots, i_{L-1}\right)$ for given $M, L$, and $\Delta$.

Definition 2. The function $\epsilon_{\mathcal{B}}(k, Z, L, \Delta)$ maps a positive integer $k$ to the sequence $\left(S_{1}, S_{2}, \ldots, S_{L}\right)$ for given $Z, L$, and $\Delta$.

Definition 3. The inverse functions of $\epsilon_{\mathcal{A}}(n, M, L, \Delta)$ and $\epsilon_{\mathcal{B}}(k, Z, L, \Delta)$ are $\epsilon_{\mathcal{A}}^{-1}\left(\left(i_{0}, i_{1}, \ldots, i_{L-1}\right), M, L, \Delta\right)$ and $\epsilon_{\mathcal{B}}^{-1}\left(\left(S_{1}, \ldots, S_{L}\right), Z, L, \Delta\right)$, respectively.

For $\epsilon_{\mathcal{A}}(n, M, L, \Delta)$, we use the expansion in (34). We first determine $i_{0}$ as the maximum value of $x$ such that $n(x) \triangleq \sum_{i_{0}=0}^{x} f\left(i_{0}\right)<n$, where $f\left(i_{0}\right) \triangleq$ $\mathcal{B}_{L, \Delta}\left(M-L+\min \left(0, \Delta-i_{0}\right)\right)$. We then obtain $\left(S_{1}, \ldots, S_{L}\right)$ by using $\epsilon_{\mathcal{B}}\left(n\left(i_{0}\right)-n, M-L, L, \Delta\right)$ for $i_{0}<\Delta$ and $\epsilon_{\mathcal{B}}\left(n\left(i_{0}\right)-n, M-L+\Delta-i_{0}, L, \Delta\right)$ for $i_{0} \geq \Delta$. We finally calculate $i_{\ell}=i_{0}+\sum_{j=1}^{\ell}\left(1+S_{j}\right)$ for $\ell \in\{1,2, \ldots, L-1\}$.

For $\epsilon_{\mathcal{B}}(k, Z, L, \Delta)$, we exploit the sum in 29) and ob- 


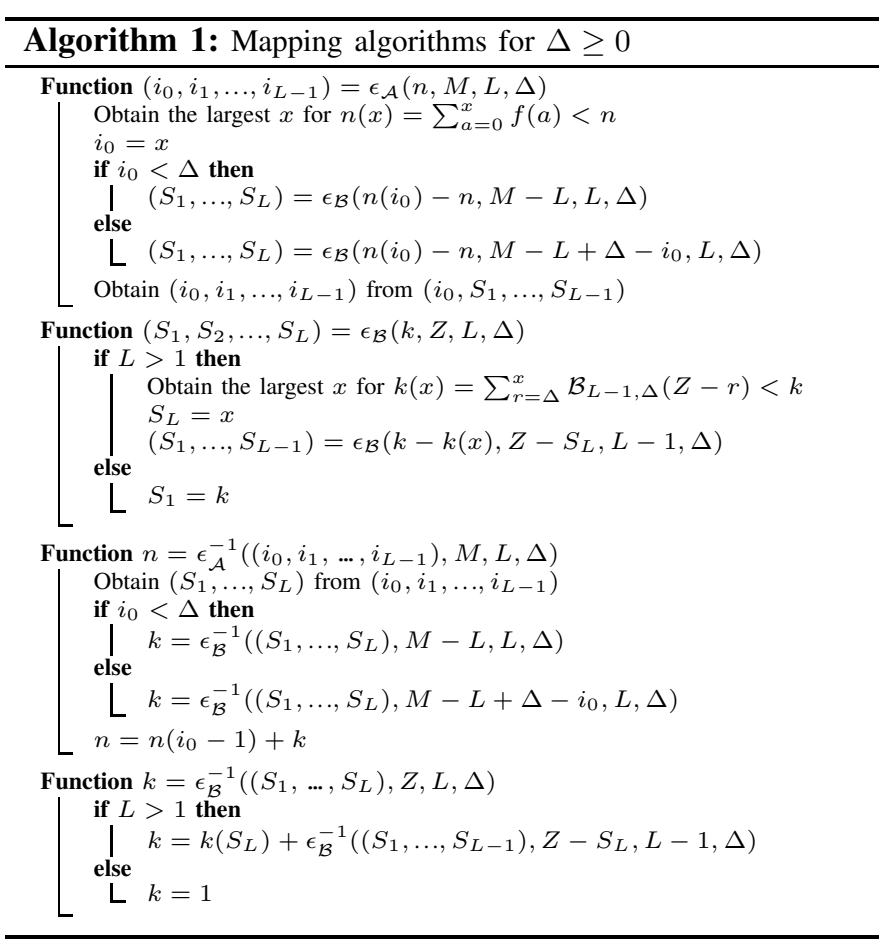

TABLE I

THE ENUMERATIONS OF $\epsilon_{\mathcal{A}}(n, M, L, \Delta)$ FOR $M=10, L=3$, AND $\Delta \in\{0,1,2\}$.

\begin{tabular}{|c|c|c|c|c|c|c|c|c|c|}
\hline & \multicolumn{3}{|c|}{$\Delta=0$} & \multicolumn{3}{|c|}{$\Delta=1$} & \multicolumn{3}{|c|}{$\Delta=2$} \\
\hline $\bar{n}$ & $i_{0}$ & $i_{1}$ & $\overline{i_{2}}$ & $i_{0}$ & $i_{1}$ & $\overline{i_{2}}$ & $i_{0}$ & $i_{1}$ & $i_{2}$ \\
\hline 1 & 0 & 8 & 9 & 0 & 6 & 8 & 0 & 4 & 7 \\
\hline 2 & 0 & 7 & 9 & 0 & 5 & 8 & 0 & 3 & 7 \\
\hline 3 & 0 & 6 & 9 & 0 & 4 & 8 & 0 & 3 & 6 \\
\hline 4 & 0 & 5 & 9 & 0 & 3 & 8 & 1 & 5 & 8 \\
\hline 5 & 0 & 4 & 9 & 0 & 2 & 8 & 1 & 4 & 8 \\
\hline 6 & 0 & 3 & 9 & 0 & 5 & 7 & 1 & 4 & 7 \\
\hline 7 & 0 & 2 & 9 & 0 & 4 & 7 & 2 & 6 & 9 \\
\hline 8 & 0 & 1 & 9 & 0 & 3 & 7 & 2 & 5 & 9 \\
\hline 9 & 0 & 7 & 8 & 0 & 2 & 7 & 2 & 5 & 8 \\
\hline 10 & 0 & 6 & 8 & 0 & 4 & 6 & 3 & 6 & 9 \\
\hline 50 & 1 & 6 & 7 & 5 & 7 & 9 & \multirow{3}{*}{\multicolumn{3}{|c|}{ N/A }} \\
\hline : & & : & & \multirow{2}{*}{\multicolumn{3}{|c|}{ N/A }} & & & \\
\hline 120 & 7 & 8 & 9 & & & & & & \\
\hline Cardinality & \multicolumn{3}{|c|}{120} & \multicolumn{3}{|c|}{50} & \multicolumn{3}{|c|}{10} \\
\hline
\end{tabular}

tain $S_{L}$ as the maximum value of $x$ such that $k(x) \triangleq$ $\sum_{r=\Delta}^{x} \mathcal{B}_{L-1, \Delta}(Z-r)<k$. Since determining $S_{L}$ reduces the original problem from $L$ parts to $L-1$ parts, where the new sum is $Z-S_{L}, \epsilon_{\mathcal{B}}(k, Z, L, \Delta)$ recalls itself as $\epsilon_{\mathcal{B}}\left(k-k(x), Z-S_{L}, L-1, \Delta\right)$ to obtain $\left(S_{1}, \ldots, S_{L-1}\right)$. This procedure is recursive and continues till $L=1$. For $L=1, \epsilon_{\mathcal{B}}(k, Z, L, \Delta)$ returns $S_{1}=k$.

The function $\epsilon_{\mathcal{A}}^{-1}\left(\left(i_{0}, i_{1}, \ldots, i_{L-1}\right), M, L, \Delta\right)$ first calculates $n\left(i_{0}-1\right)$. Afterwards, it obtains $\left(S_{1}, \ldots, S_{L-1}\right)$ from $\left(i_{0}, i_{1}, \ldots, i_{L-1}\right)$. Finally, it returns $n=n\left(i_{0}\right)+k$, where $k$ is $\epsilon_{\mathcal{B}}^{-1}\left(\left(S_{1}, \ldots, S_{L}\right), M-L, L, \Delta\right)$ for $i_{0}<\Delta$ and $\epsilon_{\mathcal{B}}^{-1}\left(\left(S_{1}, \ldots, S_{L}\right), M-L+\Delta-i_{0}, L, \Delta\right)$ for $i_{0} \geq \Delta$ based on (34).

The function $\quad \epsilon_{\mathcal{B}}^{-1}\left(\left(S_{1}, \ldots, S_{L}\right), Z, L, \Delta\right) \quad$ first calculates $k\left(S_{L}\right)$. It then returns the result as $k=k\left(S_{L}\right)+\epsilon_{\mathcal{B}}^{-1}\left(\left(S_{1}, \ldots, S_{L-1}\right), M-S_{L}, L-1, \Delta\right)$. For $L=1, \epsilon_{\mathcal{B}}^{-1}\left(\left(S_{1}, \ldots, S_{L}\right), Z, L, \Delta\right)$ is 1 .

The pseudocodes for the mapping algorithms are provided in Algorithm 1, As the closed-form expressions of $\mathcal{A}_{L, \Delta}(M)$ and $\mathcal{B}_{L, \Delta}(Z)$ are available in Theorem 2 and Lemma 1 respectively, the time complexity of these algorithms linearly scales with $M, L$, and $1 / \Delta$. The algorithms can also be efficiently implemented as they rely on recursions.

In TABLE【 we exemplify the output of $\epsilon_{\mathcal{A}}(n, M, L, \Delta)$ for $M=10, L=3$, and $\Delta \in\{0,1,2\}$. For instance, for $\Delta=2$, there are at least 2 integers between any two adjacent indices and there are 10 valid sequences. Hence, 3 information bits can be encoded by using the decimal number converted from the binary number constructed with the information bits.

3) Impact of IS on the communication receiver performance: The ML detector under the IS can be expressed as

$$
\{\hat{\boldsymbol{i}}, \hat{\boldsymbol{h}}\}=\arg \max _{\substack{i_{\ell} \in\{0, \ldots, M-1\} \\ h_{\ell} \in \mathbb{Z}_{H} \\ i_{p}<i_{r} \text { for } p<r \\ S_{q} \geq \Delta \text { for } q \in 1, \ldots, L}} \Re\left\{\sum_{\ell=0}^{L-1} \tilde{d}_{\dot{i}_{\ell}} \mathrm{e}^{-\mathrm{j} 2 \pi \dot{h}_{\ell} / H}\right\},
$$

where the condition $S_{q} \geq \Delta$ reduces the search space. A low-complexity receiver based on (35) can be implemented as follows:

- Obtain $\left\{\hat{i}_{0}, \hat{h}_{0}\right\}$ that maximizes $\hat{d}_{l, z}$ for $l \in\{0,1, \ldots, M$ 1\} and $z \in \mathbb{Z}_{H}$.

- Calculate $\left\{\hat{i}_{\ell}, \hat{h}_{\ell}\right\}$ that maximizes $\hat{d}_{l, z}$ for $l \in$ $\{0,1, \ldots, M-1\}$ and $z \in \mathbb{Z}_{H}$ such that $\min \left(\mid \hat{i}_{\ell}-\right.$ $\left.\hat{i}_{\ell^{\prime}}|, M-| \hat{i}_{\ell}-\hat{i}_{\ell^{\prime}} \mid\right) \geq \Delta+1$ for all $0 \leq \ell^{\prime} \leq \ell-1$ till detecting the $(L-1)$ th index and the corresponding PSK symbol.

- Re-order the detected chirp and PSK symbol indices such that $\hat{i}_{p}<\hat{i}_{r}$ for $p<r$.

Note that the IS can slightly decrease the error rate since it restricts the valid index combinations.

\section{B. Solution \#2: Range Estimation over LMMSE Channel Estimate}

For this solution, we remove the impact of the waveform on the range estimation by using the LMMSE estimate of $\mathbf{h}$, i.e., $\tilde{\mathbf{h}}=\mathbf{W}^{\mathrm{H}}\left(\mathbf{W} \mathbf{W}^{\mathrm{H}}+\sigma_{\mathrm{n}}^{2} \mathbf{I}\right)^{-1} \mathbf{b}$. For a single target, the estimate of $\tilde{\tau}_{1}$ can then be obtained as

$$
\tilde{\tau}_{1}=\arg \max _{\dot{\tau}_{1}}\left|\Re\left\{\mathbf{t}_{\dot{\tau}_{1}}{ }^{\mathrm{H}} \mathbf{W}^{\mathrm{H}}\left(\mathbf{W} \mathbf{W}^{\mathrm{H}}+\sigma_{\mathrm{n}}^{2} \mathbf{I}\right)^{-1} \mathbf{b}\right\}\right|,
$$

where $\tilde{\alpha}_{1}=\Re\left\{\mathbf{t}_{\tilde{\tau}_{1}}{ }^{\mathrm{H}} \mathbf{W}^{\mathrm{H}} \mathbf{b}\right\} /\left(\mathbf{w}^{\mathrm{H}} \mathbf{w}+\sigma_{\mathrm{n}}^{2}\right)$. For multiple targets, we also consider the iterative procedure in (27) and (28). The main disadvantage of this method is that it does not attain the CRLB of ranges if the waveform in the frequency domain is not unimodular as demonstrated in Section $\mathrm{V}$. In addition, it requires an accurate estimation of the noise variance. 


\section{CRLB for Range and Reflection Coefficients}

To derive CRLB for range and reflection coefficients, we follow a similar approach proposed in [42]. We first re-express $b_{k}$ as

$$
\begin{aligned}
b_{k}=\zeta_{k} w_{k}+\eta_{k} & =\sum_{s=1}^{R} \alpha_{s}\left|w_{k}\right| \mathrm{e}^{-\mathrm{j} 2 \pi\left(f_{\mathrm{c}}+\frac{k}{T_{\mathrm{s}}}\right) \tau_{s}+\mathrm{j} \angle w_{k}}+\eta_{k} \\
& =\sum_{s=1}^{R} \mu_{k s}+\mathrm{j} \nu_{k s}+\eta_{k}
\end{aligned}
$$

where $\mu_{k s}=\alpha_{s}\left|w_{k}\right| \cos \left(-2 \pi\left(f_{\mathrm{c}}+\frac{k}{T_{\mathrm{s}}}\right) \tau_{s}+\angle w_{k}\right)$ and $\nu_{k s}=$ $\alpha_{s}\left|w_{k}\right| \sin \left(-2 \pi\left(f_{\mathrm{c}}+\frac{k}{T_{\mathrm{s}}}\right) \tau_{s}+\angle w_{k}\right)$. Let $\mathbf{p}$ be the vector that contains the unknown parameters as $\mathbf{p}=\left[p_{1}, \ldots, p_{2 R}\right]=$ $\left[\tau_{1}, \ldots, \tau_{R}, \alpha_{1}, \ldots, \alpha_{R}\right]$. The element on the $i$ th row and $j$ th column of the $2 R \times 2 R$ Fisher information matrix (FIM) can then be calculated as $J_{i j}=\frac{2}{\sigma_{\mathrm{n}}^{2}} \sum_{s=1}^{R} \sum_{k=L_{\mathrm{d}}}^{L_{\mathrm{u}}} \frac{\partial \mu_{k s}}{\partial p_{i}} \frac{\partial \mu_{k s}}{\partial p_{j}}+$ $\frac{\partial \nu_{k s}}{\partial p_{i}} \frac{\partial \nu_{k s}}{\partial p_{j}}$. By evaluating the derivatives, $J_{i j}$ can be obtained as

$$
J_{i j}= \begin{cases}\frac{8 \pi^{2} \alpha_{i}^{2}}{\sigma_{2}^{2}} \sum_{k=L_{\mathrm{d}}}^{L_{\mathrm{u}}}\left|w_{k}\right|^{2}\left(\frac{k}{T_{\mathrm{s}}}+f_{\mathrm{c}}\right)^{2}, & i=j \leq R \\ \frac{2 \alpha_{i-R}^{2}}{\sigma_{\mathrm{n}}^{2}} \sum_{k=L_{\mathrm{d}}}^{L_{\mathrm{u}}}\left|w_{k}\right|^{2}, & i=j>R \\ 0 & \text { otherwise }\end{cases}
$$

The CRLBs for the unknown parameters are the diagonal elements of the inverse of the FIM. Since the FIM is a diagonal matrix, the unbiased CRLB of the ranges and the CRLB of the reflection coefficients are given by

$$
\begin{aligned}
\sigma_{\text {range }}^{2} & \triangleq \mathbb{E}\left\{\sum_{s=1}^{R}\left|r_{s}-\tilde{r}_{s}\right|^{2}\right\} \geq \frac{c^{2}}{4} \sum_{i=1}^{R} \frac{1}{J_{i i}} \\
& =\frac{\sigma_{\mathrm{n}}^{2} c^{2}}{32 \pi^{2} \sum_{k=L_{\mathrm{d}}}^{L_{\mathrm{u}}}\left|w_{k}\right|^{2}\left(\frac{k}{T_{\mathrm{s}}}+f_{\mathrm{c}}\right)^{2}} \sum_{s=1}^{R} \frac{1}{\alpha_{s}^{2}},
\end{aligned}
$$

and

$$
\begin{aligned}
\sigma_{\text {coeff }}^{2} & \triangleq \mathbb{E}\left\{\sum_{s=1}^{R}\left|\alpha_{s}-\tilde{\alpha}_{s}\right|^{2}\right\} \geq \sum_{i=R+1}^{2 R} \frac{1}{J_{i i}} \\
& =\frac{\sigma_{\mathrm{n}}^{2}}{2 \sum_{k=L_{\mathrm{d}}}^{L_{\mathrm{u}}}\left|w_{k}\right|^{2}} \sum_{s=1}^{R} \frac{1}{\alpha_{s}^{2}},
\end{aligned}
$$

respectively. By using the fact that $\mathbb{E}\left\{\left|w_{k}\right|^{2}\right\}=\left|f_{k}\right|^{2}, J_{i j}$ can be re-expressed by replacing $\left|w_{k}\right|^{2}$ with $\left|f_{k}\right|^{2}$ in (38). Therefore, (39) and (40) can be modified by replacing $\left|w_{k}\right|^{2}$ with $\left|f_{k}\right|^{2}$.

In the literature, various CRLBs are derived for different scenarios. For instance, by using OFDM with unimodular sequences, the CRLB of $\sigma_{\text {range }}^{2}$ was calculated in [43, Section 3.3.3] as

$$
\sigma_{\text {range }}^{2} \geq \frac{3 \sigma_{\mathrm{n}}^{2} c^{2}}{8 \pi^{2} M\left(M^{2}-1\right)} \sum_{s=1}^{R} \frac{1}{\alpha_{s}^{2}} .
$$

In [18], it was derived when the OFDM subcarriers are weighted. The main difference between (39) and the CRLBs derived in these studies is the distance-dependent phase in the channel model. While these studies assume that the phase is unknown and independent from the target's location, we

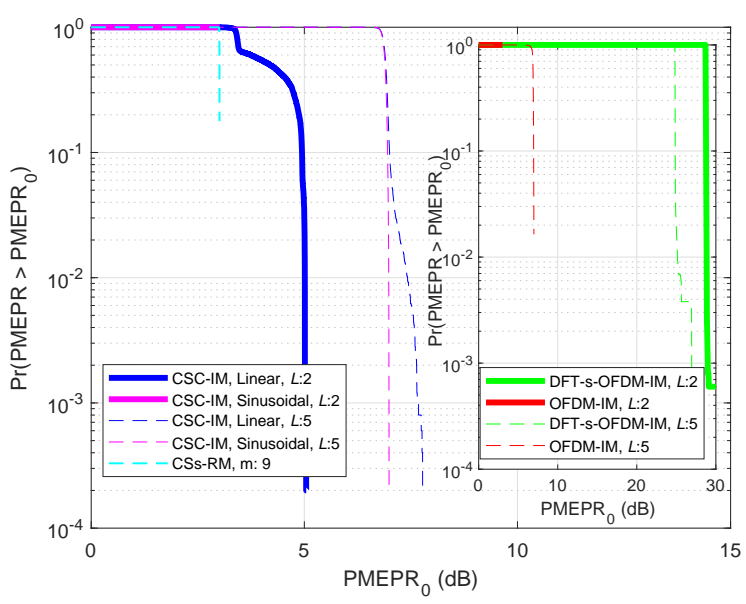

Fig. 6. PMEPR distributions for different schemes.

consider the fact that the phase is a function of the target's distance in (7) [44].

\section{NUMERICAL RESULTS}

We consider IEEE 802.11ay OFDM with 4 channels, where $T_{\mathrm{s}} \approx 194 \mathrm{~ns}$ and $T_{\mathrm{CP}} \approx 48.48 \mathrm{~ns}, f_{\mathrm{c}}=64.8 \mathrm{GHz}$, $f_{\text {sample }}=10.56 \mathrm{Gsps}, N=2048$, and $N_{\mathrm{CP}}=512$. We assume that $L_{\mathrm{u}}=724, L_{\mathrm{d}}=-723$, and $D=1382$, and $M=1536$ for 4 channel 3 . Therefore, the bandwidth of the signal is approximately $7.2 \mathrm{GHz}$ for CSC-IM. The maximum range of the radar is $7.27 \mathrm{~m}$. The modulation symbols are based on QPSK, i.e., $H=4$. The FDSS coefficients are chosen based on (4) and (5) and assumed to be known at the RXc.We consider $L \in\{1,2,5\}$ and set $\Delta$ to 84 (i.e., no bits loss based on Fig. 5(b) for $L=2$ and 252 (i.e., 10 bits are sacrificed based on Fig. 5(a) for $L=5$, when the IS is considered. Otherwise, $\Delta$ is set to 0 . We compare the proposed scheme with three different alternatives: OFDM-IM, DFT-s-OFDM-IM (i.e., no FDSS is applied), and the CSs-RM [11], [12]. For OFDM-IM, an ML detector that incorporates the channel frequency response is utilized [27]. For CSs-RM, we use a seed GCP of length $N=3$ and use $m=9$. To facilitate the ML-based decoder proposed in [12] for CSs, we consider only $(m-1)$ ! cosets. Since these schemes do not use FDSS, their bandwidths are approximately $7.9 \mathrm{GHz}$. For fading channel, a power delay profile with three paths where the relative powers are $0 \mathrm{~dB},-10 \mathrm{~dB},-20 \mathrm{~dB}$ at $0 \mathrm{~ns}, 10 \mathrm{~ns}$, and $20 \mathrm{~ns}$ with Rician factors of 10,0 , and 0 , respectively, is considered. The number of information bits transmitted are 12 , 24, and 56 bits for IM-based schemes without IS for $L=1,2$, and 5 , respectively. When IS is considered, 24 bits (i.e., no SE loss due to the IS) and 46 bits (i.e., SE loss due to the IS) are transmitted for $L=2$ and 5, respectively. With CSs-RM, 35 bits are transmitted for each OFDM symbol.

\footnotetext{
${ }^{3}$ The reason for $M=1536$ is that we can compare the proposed scheme with the CSs-RM under this configuration.
} 


\section{A. Communications Performance}

In Fig. 6, we compare PMEPR distributions. The signals are over-sampled to measure PMEPR accurately. The PMEPR is always less than or equal to $3 \mathrm{~dB}$ for CSs-RM and sinusoidal chirps for $L=2$. However, the distortion on linear chirps due to the truncation is higher than the one for sinusoidal chirps. Therefore, the CSs are not accurately formed with linear chirps under our simulation settings and the maximum PMEPR reaches to $5 \mathrm{~dB}$. For $L=5$, the PMEPR is still limited for the proposed scheme and the maximum PMEPRs are $10 \log _{10} 5=6.98 \mathrm{~dB}$ and $7.5 \mathrm{~dB}$ for sinusoidal and linear chirps, respectively. On the other hand, they result in a higher SE as compared to CSs-RM. OFDM-IM results in PMEPR distributions similar to the ones for the proposed scheme for $L=2$ and $L=5$. However, it does not spread the energy in the frequency domain, which is needed for radar functionality. While DFT-s-OFDM-IM spreads the energy in time, it causes signals with very high PMEPRs. The main reason for this behavior is that DFT-s-OFDM-IM actives only $L$ indices that are represented as Dirichlet-sinc pulses in time (see Fig. 5 in [2]). Therefore, CSC-IM is superior to DFT-s-OFDM-IM and OFDM-IM for radar applications by reducing PMEPR and spreading the energy in both time and frequency.

In Fig. 7] we compare the BLER performance in AWGN and fading channel. For the proposed scheme, the receiver equalizes the signal even in the AWGN channel because of FDSS. Since a flatter response improves the BLER result in both AWGN and fading channels [10], we consider only linear CSCs for the rest of the analysis. In Fig. 7(a) we provide BLER comparisons for a given $E_{\mathrm{b}} / N_{0}$. The CSs-RM is superior to all schemes and provides $1-2 \mathrm{~dB}$ gain at $1 \mathrm{e}-3$ BLER. The CSC-IM operates in the range of $3-4 \mathrm{~dB} E_{\mathrm{b}} / N_{0}$ at 1e-3 BLER and the performance degrades slightly for a larger $L$. Their error rates are similar to those of OFDM-IM and DFT-s-OFDM-IM, which shows the equalization due to the FDSS do not degrade the error rate under our simulation

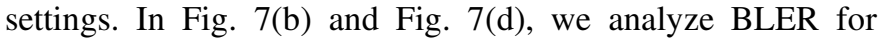
a given SNR. The BLERs for CSC-IM and orthogonal IM schemes (i.e., OFDM-IM and DFT-s-OFDM-IM) approach to the corresponding UBs given in (15), rapidly. As opposed to CSs-RM, the proposed scheme provides a range of solutions with the various data rates, maximum PMEPRs, and operating SNR points. For example, for $L=1$, it results in $0 \mathrm{~dB}$ PMEPR with a very low data rate transmission while it increases the data rate by using $L=5$ chirps at the expense of a higher PMEPR. In Fig. [7/(c) and Fig. Z7(d), we analyze the same schemes in fading channel. The performance of OFDM-IM is worse than all other schemes since it does not exploit the frequency selectivity. The slopes of the BLER curves for CSC-IM under the fading channel are also noticeably higher than the ones for OFDM-IM. Although the DFT-s-OFDM-IM is similar to CSC-IM, a larger power back-off is required for DFT-s-OFDM-IM (see PMEPR distributions in Fig. 6). We also observe that the difference between CSs-RM and CSC-IM diminishes in the fading channel and it is less than $1 \mathrm{~dB}$ for $L=2$. It is worth noting that the ML detector for CSs-RM is based on an ML-based algorithm [12], which causes a high- complexity detector due to the second-order coset term. On the other hand, the proposed scheme relies on a single $M$-IDFT, per-bin ML detection, and recursive mapping rules discussed in Section IV-A2, We also observe that the IS slightly reduces the error rate as in Fig. 7[(d) as IS limits the search space for indices as discussed in Section IV-A3.

\section{B. Radar Performance}

We consider two scenarios for evaluating RXr performance. In the first scenario, a single target is assumed. Its location is drawn from a uniform distribution between $2 \mathrm{~m}$ and $3 \mathrm{~m}$ and the true value of the reflection coefficient is set to -1 , which considers the phase change of a reflected signal [44]. For the second scenario, we consider two targets located nearby. The location of the first target is random between $2 \mathrm{~m}$ and $3 \mathrm{~m}$ and its reflection coefficient, unknown to the $\mathrm{RXr}$, is set to $-\sqrt{2} / 2$. The second target with the true value of the reflection coefficient of $-\sqrt{2} / 2$ is away from the first target by $\Delta r$, where $\Delta r$ is a randomly chosen between $1.5 r_{\text {min }} \approx 3.16 \mathrm{~cm}$ and $2 r_{\min } \approx 4.21 \mathrm{~cm}$ for $r_{\min } \approx 2.1 \mathrm{~cm}$. We consider the proposed scheme with linear chirps and compare it with CSs-RM. We exclude OFDM-IM (as it does not distribute the signal energy in the frequency domain) and DFT-s-OFDM-IM (as it causes high PMEPR) for radar functionality.

In Fig. 8, we evaluate the accuracy of range estimators by providing RMSE versus SNR curves. In Fig. \&[a) and g(b) we consider the first scenario. The performance of schemes are very similar to each other. The MF-based estimator attains the CRLB derived in (39) as shown in Fig. (8)(a), For the sake of comparison, we also plot the CRLB in (41) for the case when the phase information is unknown and not a function of the target location. The difference between these two bounds indicates the phase information has a notable impact on the accuracy, which can be exploited at high SNR, i.e., for strong reflections. For the LMMSE-based estimator, the bound is only attained for CSs-RM. This is because CSs are unimodular in the frequency domain, while the symbol energy is not distributed identically to the frequency bins for linear chirps due to the multiple CSCs and FDSS. In Fig. \& (c) and Fig. $8(d)$ we consider the second scenario. Since RXr estimate targets' locations by using the sequence in the frequency domain, the waveform characteristics in the frequency domain plays a role in the accuracy. For example, the CSs-RM are the most prominent ones as it leads to sequences based on QPSK. The subcarriers are populated with arbitrary complex numbers for CSC-IM, which degrades the accuracy slightly. When IS is not adopted for ML-based estimation, Fig. E[(c) shows CSC-IM saturates and never attains the corresponding CRLB. However, when IS is utilized, the accuracy of CSC-IM with $L=2,5$ is similar to CSC-IM with $L=1$ and attains the CRLB. For the LMMSE-based estimator, CRLB is not attained for CSC-IM (due to the FDSS with non-unimodular coefficients for CSCs) while it is achieved with CSs-RM as in Fig. E[(d) This result implies that using unimodular sequences is beneficial for radar as it yields superior results with both LMMSE and MF-based estimators. However, the price paid is a higher-complexity $\mathrm{RXr}$ for decoding CSs-RM. Although it is possible to use a 


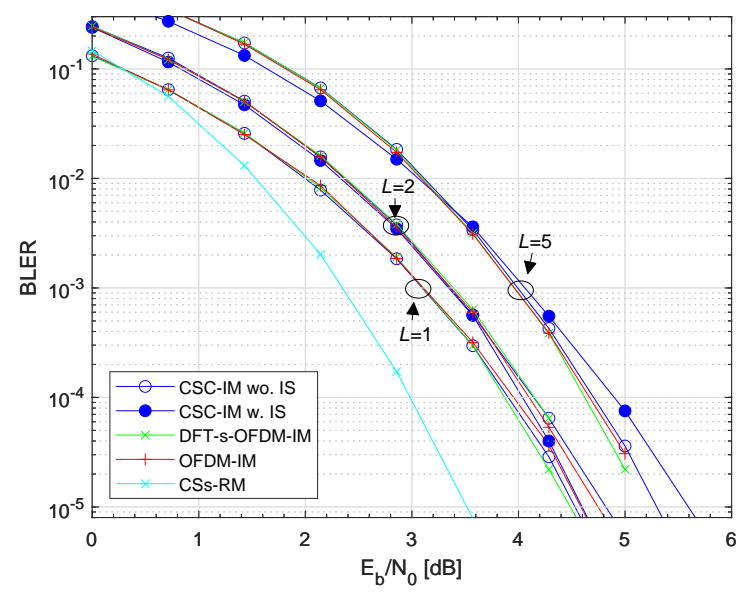

(a) BLER versus $E_{\mathrm{b}} / N_{0}$ in AWGN.

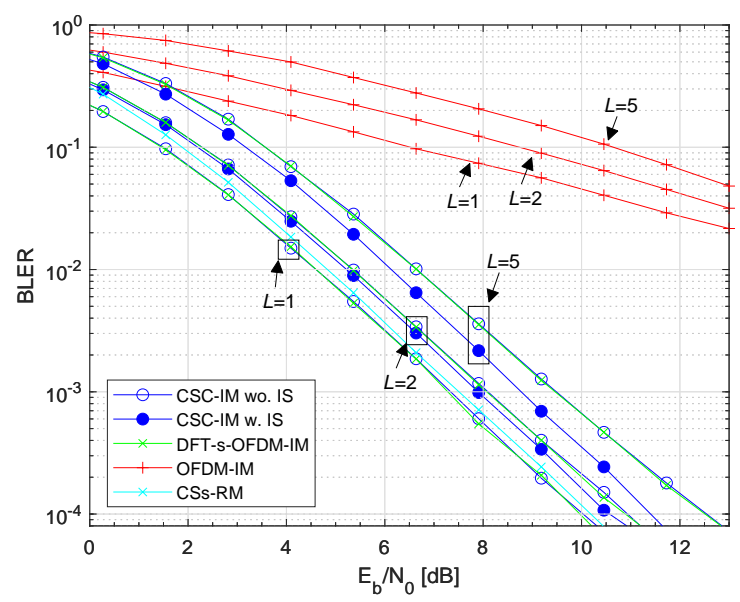

(c) BLER versus $E_{\mathrm{b}} / N_{0}$ in fading channel.

Fig. 7. BLER performance at RXr for different schemes.

lower-complexity code with QPSK, it is challenging to address the high PMEPR for OFDM. From this aspect, CSs-RM is promising for OFDM-based DFRC applications. On the other hand, CSC-IM is more flexible in terms of size and data rate as compared to CSs-RM.

In Fig. 9, we analyze the resolution for the aforementioned schemes by sweeping the distance between two targets. We fix the SNR at $20 \mathrm{~dB}$. All schemes resolve the targets after the minimum resolution $r_{\min }=2.1 \mathrm{~cm}$. On the other hand, CSC-IM without IS cannot resolve the targets as accurate as CSC-IM with IS even the distance between targets is larger than $r_{\min }$. The results are in line with the ones as observed in Fig. 8 For the LMMSE-based estimation, they do not attain the CRLB except for the CSs-RM although the accuracy improves after $r_{\min }$.

\section{CONCLUding REMARKS}

In this study, we propose CSC-IM for DFRC scenarios. We show that this scheme can generate wideband signals while bringing a trade-off between SE and maximum PMEPR, i.e., the SE increases with the number of CSCs at the expense of a

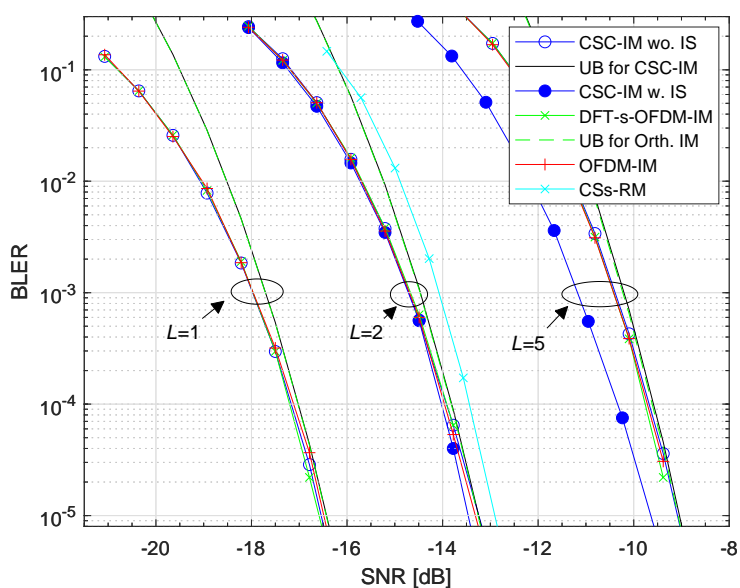

(b) BLER versus SNR in AWGN.

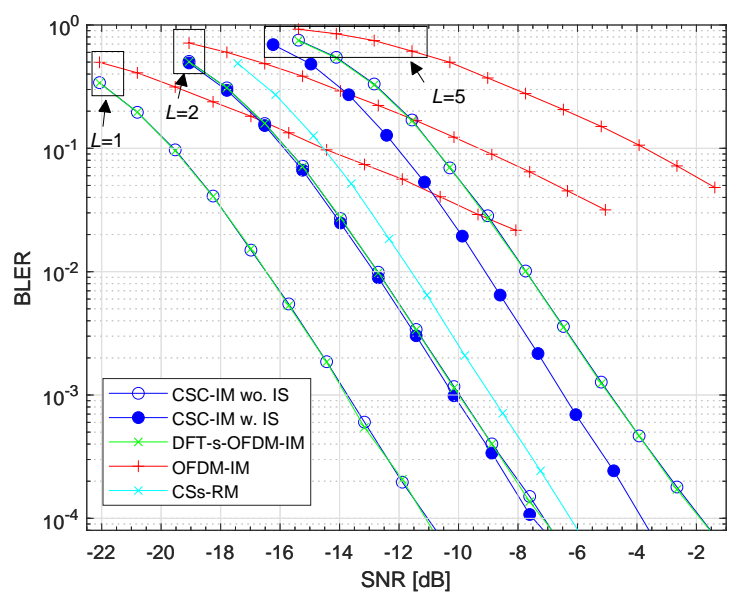

(d) BLER versus SNR in fading channel.

higher maximum PMEPR. As a special case, we prove that the transmitted signals in the frequency domain lead to new CSs based on chirps. We also exemplify that Bessel functions and Fresnel integrals can be useful for generating GCPs. CSC-IM is more flexible than the standard CSs based on RM codes in the sense that it allows one to control the maximum PMEPR theoretically for the sake of increasing the SE while being more flexible in terms of the number of utilized subcarriers. Besides, since CSC-IM does not utilize a coset term needed for the CSs-RM, it enjoys a low-complexity decoder. In this study, we derive the UB of the BLER for CSC-IM, which also captures the analysis for OFDM-IM and DFT-s-OFDM-IM. With comprehensive simulations, we show that the CSC-IM offers a lower PMEPR than DFT-s-OFDM-IM while exploiting frequency selectivity as compared to OFDM-IM. CSC-IM is more suitable for radar functionality as compared to DFT-s-OFDM-IM and OFDM-IM as it reduces the PMEPR while allowing controllable AC properties. We consider two range estimation methods: MF-based and LMMSE-based estimations. For the MF-based estimation, we introduce IS to generate a low AC zone. We investigate the impact of IS on 


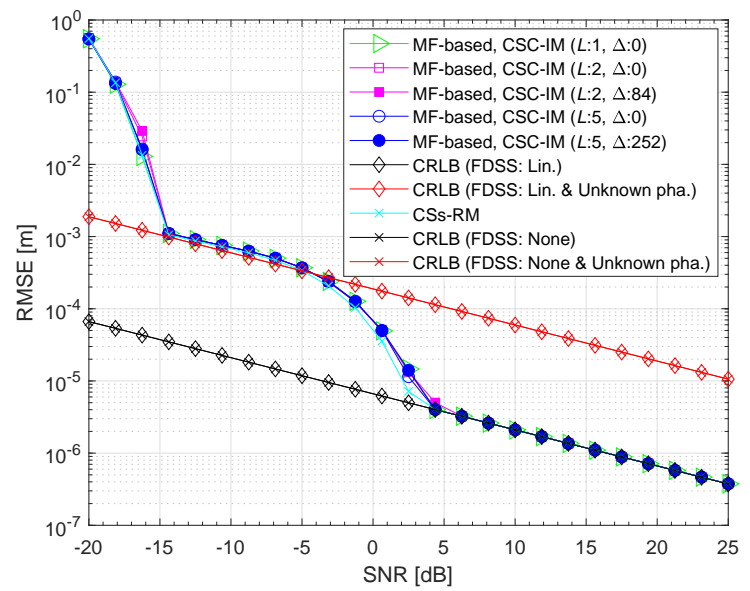

(a) MF-based estimation and a single target.

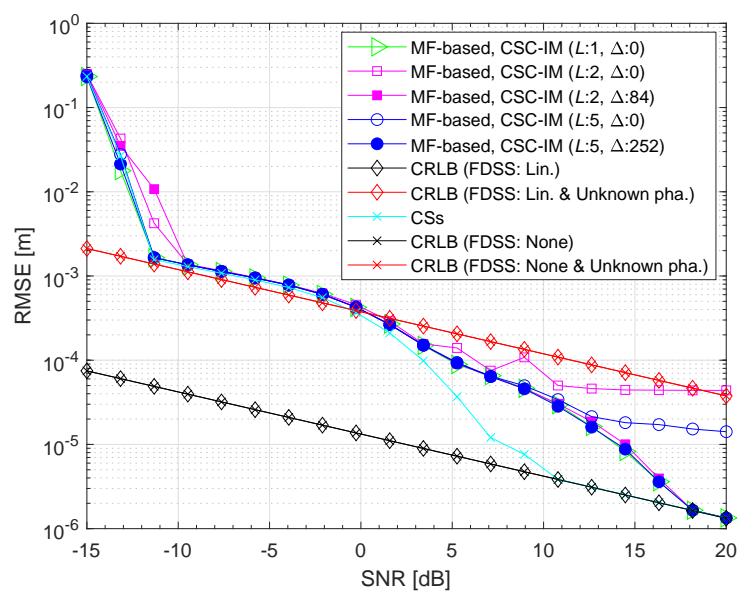

(c) MF-based estimation and two nearby targets.

Fig. 8. Accuracy analysis for different estimators and scenarios.

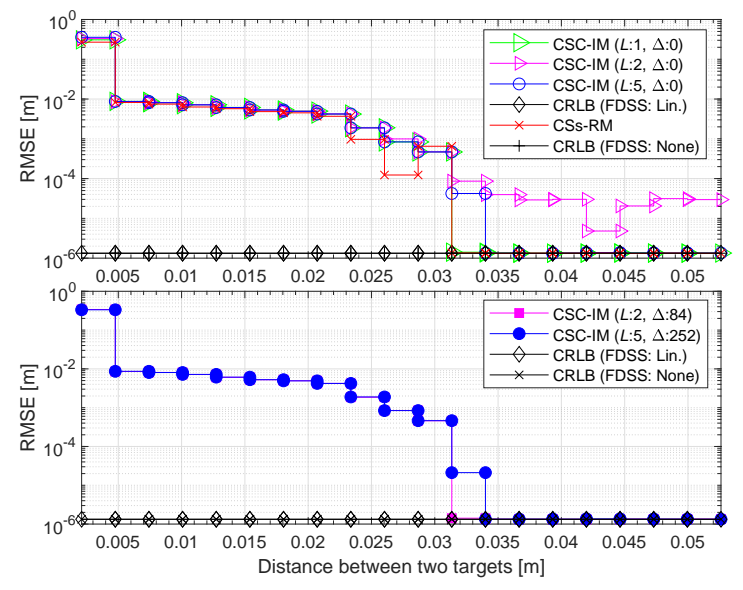

(a) ML-based estimation.

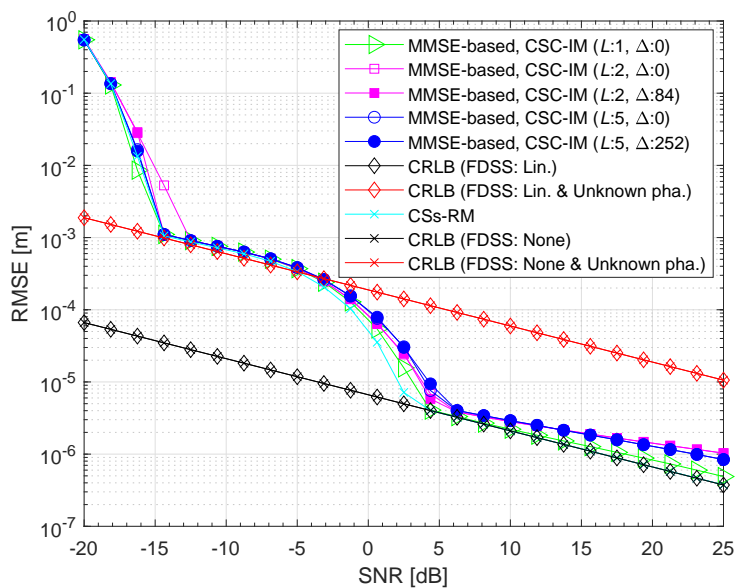

(b) LMMSE-based estimation and a single target.

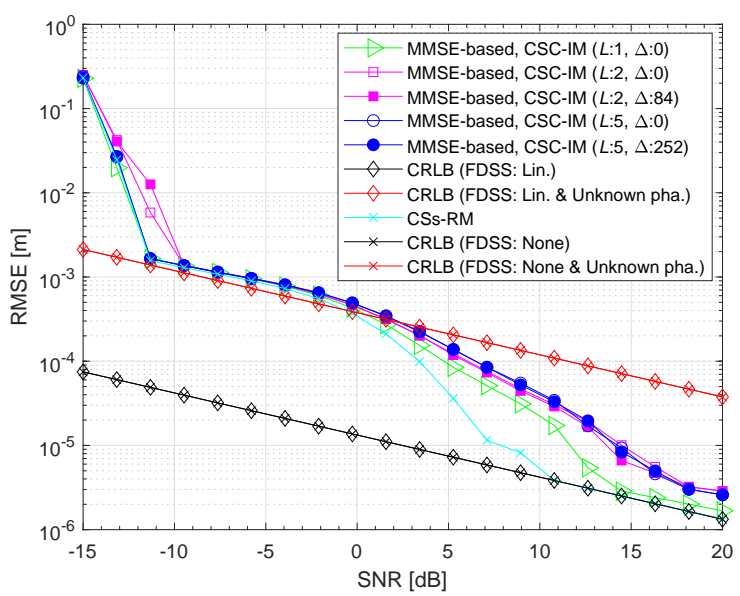

(d) LMMSE-based estimation and two nearby targets.
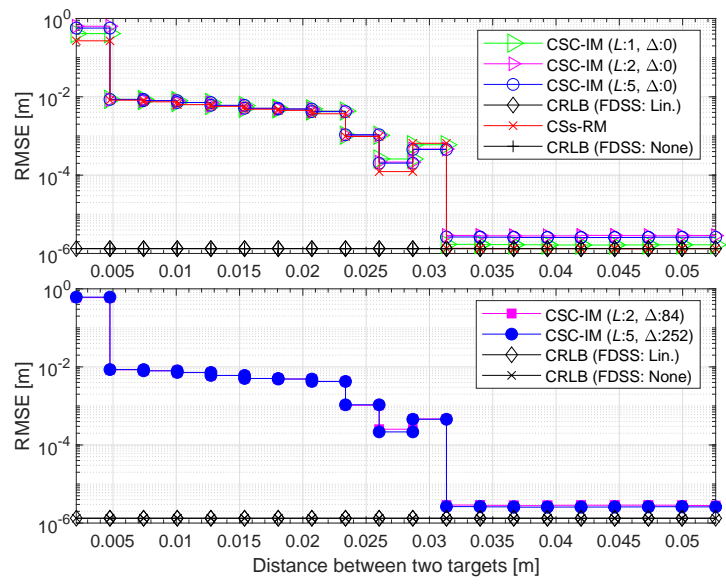

(b) LMMSE-based estimation.

Fig. 9. Resolution analysis for different estimators. 
SE and provide algorithms that map indices to information bits or vice versa. We show that IS helps the estimation accuracy to attain the corresponding CRLB.

\section{REFERENCES}

[1] S. Hoque and A. Şahin, "Index-modulated circularly-shifted chirps for dual-function radar \& communication systems," in Proc. IEEE Global Communications Conference - ISAC Workshop, Dec. 2020, pp. 1-6.

[2] S. Hoque, C.-Y. Chen, and A. Şahin, "A wideband index modulation with circularly-shifted chirps," in Proc. IEEE Consumer Commun. \& Netw. Conf. (CCNC), Jan. 2021, pp. 1-6.

[3] B. Paul, A. R. Chiriyath, and D. W. Bliss, "Survey of RF communications and sensing convergence research," IEEE Access, vol. 5, pp. 252-270, 2017

[4] D. Ma, N. Shlezinger, T. Huang, Y. Liu, and Y. C. Eldar, "Joint radarcommunication strategies for autonomous vehicles: Combining two key automotive technologies," IEEE Signal Process. Mag., vol. 37, no. 4, pp. 85-97, Jun. 2020.

[5] 3GPP, "NR; NR and NG-RAN overall description," TS 38.300 V16.6.0, Jul. 2021.

[6] — "E-UTRA and E-UTRAN; overall description," TS 36.300 V16.6.0, Jul. 2021.

[7] S. Darlington, "Pulse transmission," Patent US2 678 997A, Dec., 1949.

[8] Hao Shen and A. Papandreou-Suppappola, "Diversity and channel estimation using time-varying signals and time-frequency techniques," IEEE Trans. Signal Process., vol. 54, no. 9, pp. 3400-3413, Sep. 2006.

[9] X. Ouyang and J. Zhao, "Orthogonal chirp division multiplexing," IEEE Trans. Commun., vol. 64, no. 9, pp. 3946-3957, Sep. 2016.

[10] A. Şahin, N. Hosseini, H. Jamal, S. Hoque, and D. W. Matolak, "DFTspread-OFDM based chirp transmission," IEEE Commun. Lett., 2020.

[11] J. A. Davis and J. Jedwab, "Peak-to-mean power control in OFDM, Golay complementary sequences, and Reed-Muller codes," IEEE Trans. Inf. Theory, vol. 45, no. 7, pp. 2397-2417, Nov. 1999.

[12] A. Sahin and R. Yang, "A generic complementary sequence construction and associated encoder/decoder design," IEEE Trans. Commun., pp. 115, 2021.

[13] C. Sturm, T. Zwick, and W. Wiesbeck, "An OFDM system concept for joint radar and communications operations," in Proc. IEEE Vehicular Technology Conference (VTC), 2009, pp. 1-5.

[14] C. Sturm and W. Wiesbeck, "Waveform design and signal processing aspects for fusion of wireless communications and radar sensing," Proceedings of the IEEE, vol. 99, no. 7, pp. 1236-1259, 2011.

[15] M. Braun, C. Sturm, and F. K. Jondral, "Maximum likelihood speed and distance estimation for OFDM radar," in Proc. IEEE Radar Conference (RadarConf), 2010, pp. 256-261.

[16] M. Bică and V. Koivunen, "Delay estimation method for coexisting radar and wireless communication systems," in Proc. IEEE Radar Conference (RadarConf), 2017, pp. 1557-1561.

[17] S. Sharma, M. Bică, and V. Koivunen, "Reduced PMEPR multicarrier radar waveform design," in Proc. IEEE Asilomar Conference on Signals, Systems, and Computers, 2019, pp. 2048-2052.

[18] A. Turlapaty, Y. Jin, and Y. Xu, "Range and velocity estimation of radar targets by weighted OFDM modulation," in Proc. IEEE Radar Conference (RadarConf), 2014, pp. 1358-1362.

[19] S. D. Liyanaarachchi, C. B. Barneto, T. Riihonen, and M. Valkama, "Joint OFDM waveform design for communications and sensing convergence," in Proc. IEEE International Conference on Communications (ICC), 2020, pp. 1-6.

[20] S. Sen and A. Nehorai, "Adaptive OFDM radar for target detection in multipath scenarios," IEEE Transactions on Signal Processing, vol. 59, no. 1, pp. 78-90, 2011.

[21] P. Kumari, J. Choi, N. González-Prelcic, and R. W. Heath, "IEEE 802.11ad-based radar: An approach to joint vehicular communicationradar system," IEEE Trans. Veh. Technol., vol. 67, no. 4, pp. 3012-3027, Apr. 2018.

[22] D. Slepian, "Permutation modulation," Proceedings of the IEEE, vol. 53, no. 3, pp. 228-236, Mar. 1965.

[23] X. Cheng, M. Zhang, M. Wen, and L. Yang, "Index modulation for 5G: Striving to do more with less," IEEE Wireless Communications, vol. 25, no. 2, pp. 126-132, Apr. 2018.

[24] N. Ishikawa, S. Sugiura, and L. Hanzo, "50 years of permutation, spatial and index modulation: From classic RF to visible light communications and data storage," IEEE Communications Surveys \& Tutorials, vol. 20, no. 3, pp. 1905-1938, 2018.
[25] M. Wen, B. Zheng, K. J. Kim, M. Di Renzo, T. A. Tsiftsis, K.-C. Chen, and N. Al-Dhahir, "A survey on spatial modulation in emerging wireless systems: Research progresses and applications," IEEE Journal on Selected Areas in Communications, vol. 37, no. 9, pp. 1949-1972, Sep. 2019

[26] T. Mao, Q. Wang, Z. Wang, and S. Chen, "Novel index modulation techniques: A survey," IEEE Communications Surveys Tutorials, vol. 21, no. 1, pp. 3150-348, Jul. 2019.

[27] E. Başar, Ü. Aygölü, E. Panayırcı, and H. V. Poor, "Orthogonal frequency division multiplexing with index modulation," IEEE Trans. Signal Process., vol. 61, no. 22, pp. 5536-5549, Apr. 2013.

[28] R. Y. Mesleh, H. Haas, S. Sinanovic, C. W. Ahn, and S. Yun, "Spatial modulation," IEEE Veh. Technol., vol. 57, no. 4, pp. 2228-2241, Jul. 2008.

[29] A. K. Khandani, "Media-based modulation: A new approach to wireless transmission," in Proc. IEEE International Symposium on Information Theory (ISIT), Jul. 2013, pp. 3050-3054.

[30] E. BouDaher, A. Hassanien, E. Aboutanios, and M. G. Amin, "Towards a dual-function MIMO radar-communication system," in Proc. IEEE Radar Conference (RadarConf), 2016, pp. 1-6.

[31] D. Ma, T. Huang, N. Shlezinger, Y. Liu, X. Wang, and Y. C. Eldar, "A DFRC system based on multi-carrier agile FMCW MIMO radar for vehicular applications," in Proc. IEEE International Conference on Commun. (ICC), 2020, pp. 1-6.

[32] J. Proakis and M. Salehi, Fundamentals of Communication Systems. Pearson Education, 2013.

[33] M. Golay, "Complementary series," IRE Trans. Inf. Theory, vol. 7, no. 2, pp. 82-87, Apr. 1961

[34] M. G. Parker, K. G. Paterson, and C. Tellambura, "Golay complementary sequences," in Wiley Encyclopedia of Telecommunications, 2003.

[35] K. G. Paterson, "Generalized Reed-Muller codes and power control in OFDM modulation," IEEE Trans. Inf. Theory, vol. 46, no. 1, pp. 104120, Jan. 2000

[36] S. Gao, M. Zhang, and X. Cheng, "Precoded index modulation for multiinput multi-output ofdm," IEEE Transactions on Wireless Communications, vol. 17 , no. 1 , pp. 17-28, Oct. 2018.

[37] M. D. Nisar, H. Nottensteiner, and T. Hindelang, "On performance limits of DFT Spread OFDM systems," in Proc. IEEE Mobile and Wireless Communications Summit, Jul. 2007, pp. 1-4.

[38] E. Başar, "OFDM with index modulation using coordinate interleaving," IEEE Wireless Commun. Lett., vol. 4, no. 4, pp. 381-384, Aug. 2015.

[39] K. G. Paterson and A. E. Jones, "Efficient decoding algorithms for generalized Reed-Muller codes," IEEE Trans. Commun., vol. 48, no. 8, pp. 1272-1285, Aug. 2000.

[40] K. Schmidt and A. Finger, "Simple maximum-likelihood decoding of generalized first-order Reed-Muller codes," IEEE Commun. Lett., vol. 9, no. 10 , pp. 912-914, Oct. 2005.

[41] A. Sahin and R. Yang, "An uplink control channel design with complementary sequences for unlicensed bands," IEEE Trans. Wireless Commun., vol. 19, no. 10, pp. 6858-6870, Jul. 2020.

[42] D. Rife and R. Boorstyn, "Single tone parameter estimation from discrete-time observations," IEEE Transactions on Information Theory, vol. 20, no. 5, pp. 591-598, Sep. 1974.

[43] M. Braun, "OFDM radar algorithms in mobile communication networks," Ph.D. dissertation, Karlsruher Institut fur Technologie, 2014.

[44] D. Tse and P. Viswanath, Fundamentals of Wireless Communication. Cambridge University Press, 2005. 